\title{
Reproduction and recruitment of corals: comparisons among the Caribbean, the Tropical Pacific, and the Red Sea*
}

\author{
Robert H. Richmond, Cynthia L. Hunter \\ Marine Laboratory, University of Guam, UOG Station, Mangilao, Guam 96923, USA
}

\begin{abstract}
Detailed reproductive data are now available for 210 of the ca 600 identified scleractinian reef coral species. The majority (131 species) are hermaphroditic broadcast spawners, although hermaphroditic brooders (11 species), gonochoristic broadcasters ( 37 species), and gonochoristic brooders $(7$ species) have also been reported. Characteristics of sexuality and mode of reproduction are generally conservative within species, genera, and even families, although some exceptions occur. Variation in timing or mode of reproduction in allopatric populations may represent adaptations to local environmental conditions or indicate problems in the taxonomy of some groups. Synchronous spawning of numerous species occurs on the Great Barrier Reef, while asynchrony among and within species has been observed in the Red Sea, Caribbean, Central Pacific, Hawaii, and southern Japan. Sexual reproduction is the primary means for successful recruitment for some coral populations, while asexual processes may be the dominant or sole means of recruitment for these same species at the limits of their ranges. Recruitment success of different reproductive strategies may vary within and between localities, and is mediated by both biotic (predation, competition) and abiotic (environmental variability, disturbancel factors. Data on reproductive patterns and recruitment success may be applied to coral reef management practices.
\end{abstract}

\section{INTRODUCTION}

Until the last decade, the majority of data on coral reproduction were anecdotal and incomplete observations based on short-term and sporadic studies (see review by Fadlallah 1983). This situation is not surprising, in light of the remoteness of tropical coral reefs from most universities and research facilities, and the logistical difficulties of studying corals in situ. However, a number of recent investigations have been published based on continuous monitoring of field populations, as well as histological and laboratory examination of individuals. Previous generalizations

\footnotetext{
- This review originates from a UNESCO/COMAR workshop held in Fiji comparing Atlantic and Pacific tropical coastal ecosystems
}

and perceived trends may now be re-examined, as data for a greater number of species over a wide geographic range have become available.

Detailed reproductive data have been reported for ca $40 \%$ of the known species from the tropical Pacific (studies from the Great Barrier Reef, Guam, Palau, Enewetak, Hawaii, Okinawa, and Panama), 30\% of Caribbean coral species, and $6 \%$ of Red Sea species. These studies provide information on coral sex (hermaphroditism vs gonochorism), mode of reproduction (brooding vs broadcast spawning), and timing of reproduction (seasonality, periodicity, and synchrony). Certain patterns of reproduction and recruitment are now discernible from these data. In this paper, we review data for 210 scleractinian species, and compare reproductive processes observed in the Caribbean, eastern Pacific, Hawaii, Central Pacific, southern Japan, Great Barrier Reef, and Red Sea. As data for more taxa and 
regions become available, additional (or different) patterns and trends may emerge.

The study of coral reproduction has advanced through numerous theses and dissertations over the last $10 \mathrm{yr}$, as well as from concerted group efforts, most notably on the Great Barrier Reef of Australia. The major pattern that has developed from the Great Barrier Reef studies is one of remarkable similarity and synchrony of reproductive activity among coral species. The majority ( $90 \%$ ) of species studied there broadcast spawn gametes annually, during the week following the full moon in the austral spring (Harrison et al. 1984, Willis et al. 1985, Babcock et al. 1986). Data from other regions (the Caribbean, Red Sea, Central Pacific, Hawaii, and southern Japan) show different patterns, with considerable variation in mode, timing, and synchrony among species. In addition, populations of the same species reported from 2 or more regions may display different reproductive traits. Globally, corals display great plasticity in their life history characteristics. These data are summarized for each region in Table 1, and across regions in Tables 2, 3 and 4 .

\section{REPRODUCTION IN GENERAL}

Corals reproduce both sexually and asexually. Sexual reproduction involves the process of gametogenesis, which may require from a few weeks for sperm, to over $10 \mathrm{mo}$ for eggs. Spawning and subsequent fertilization of eggs by sperm results in small, presumably genetically unique, dispersive propagules (planula larvae) which may settle, metamorphose and develop into primary polyps. Asexual reproduction is also common in many scleractinian species, and may occur through fragmentation (see review by Highsmith 1982), polyp bail-out (Goreau \& Goreau 1959, Sammarco 1982), or asexual production of planulae (Stoddart 1983). Asexual processes result in clonal propagules (genetic replicates of adult colonies) which, if derived from fragments, have the apparent advantages of large size and locally adapted genotypes.

\section{Sexual reproduction in corals}

\section{Hermaphroditism vs gonochorism}

In hermaphroditic species, ovaries and spermaries may develop on the same mesentery (most favids and mussids), on different mesenteries within the same polyp (most pocilloporids and acroporids; see Fig. 1), in different polyps within the same colony (e.g. Cladopsammia rolandi; de Lacaze-Duthiers 1897 in Fadlallah 1983), or at different times within the same colony (e.g
Stylophora pistillata; Loya 1976, Rinkevich \& Loya 1979a).

Corals can be simultaneous or sequential hermaphrodites (see discussion in Fadlallah 1983). Two species, Stylophora pistillata and Goniastrea favulus, exhibit protandrous development (Rinkevich \& Loya 1979b, Kojis \& Quinn 1981a). The only report (Duerden 1902) of true protogyny, in which the colony functions first as a female and becomes hermaphroditic in subsequent years, has been questioned (Szmant 1986). Most hermaphroditic corals exhibit annual protogyny, where eggs develop prior to spermary formation during a reproductive season.

Mixed breeding systems have been described for a brooding species, Porites astreoides, in which $26 \%$ of colonies examined were hermaphroditic, $28 \%$ had only female gonads, and $46 \%$ were sterile (Chornesky \& Peters 1987). In Galaxea fascicularis, some colonies are female and some are hermaphroditic, but eggs in the latter apparently serve only to provide buoyancy for the sperm packets (Heyward et al. 1987. Harrison 1989).

Hermaphroditism is advantageous when the probability of finding ( $O r$, in the case of sedentary corals, proximity to) members of the opposite sex is low, and self-fertilization is possible. Heyward \& Babcock (1986) found varying levels of success in self-crosses in 4 coral species ( $0 \%$ in Montipora digitata, 1.5 to $16 \%$ in Acropora tenuis and Goniastrea aspera, and 26 to $89 \%$ in Goniastrea favulus). In experiments performed during spawning events on Guam during the summers of 1987 and 1988, no viable planulae developed from mixing gametes from the same individuals in Acropora irregularis or A. humilis, while nearly $100 \%$ of the embryos resulting from self-fertilized eggs of Acropora tenuis developed successfully (Richmond 1989, unpubl.). Barriers to self-fertilization apparently break down with time after spawning for some species, but not for others (Heyward \& Babcock 1986, Richmond unpubl.)

Numbers of gonochoristic and hermaphroditic species within each region are summarized in Table 2. Within regions, hermaphrodites range from 60 to $100 \%$ of reported species. Globally, the majority (68\%) of coral species studied are hermaphroditic (Table 3 )

\section{Brooding vs spawning}

Fertilization may take place within the maternal polyp (brooding), or externally in the water column after gametes are shed (broadcast spawning). Species which broadcast spawn outnumber brooders in the Pacific regions and the Red Sea (Table 2). However, brooding may be the predominant mode of reproduction in the Caribbean. Overall, for the present data 
Table 1. Reproductive characteristics of corals from the Caribbean Sea, Great Barrier Reef, Central Pacific, Hawaii, Okinawa, eastern Pacific, and Red Sea. Symbols - Sex: H, hermaphroditic; G, gonochoric; x, unknown. Mode: S, spawner; B, brooder; - possibly sterile. Timing: month and lunar day of gamete release (spawners) or planulation (brooders) [month is divided into phases: 1, new moon, 3, first quarter, 5, full moon, 7, last quarter; 2, 4, 6 and 8 indicate intermediate lunar phases (after Shlesinger \& Loya 1985)]; $w$, winter; sp, spring; sr, summer; f, fall; yr, year-round; $x$, unknown

\begin{tabular}{|c|c|c|c|c|}
\hline Species & Sex & Mode & Timing & Source \\
\hline \multicolumn{5}{|l|}{ Caribbean } \\
\hline \multicolumn{5}{|l|}{ ACROPORIDAE } \\
\hline Acroporä cervicornis & $\mathrm{H}$ & $\mathrm{s}$ & Jul/Aug, 6-7 & Szmant-Froelich (1984), Szmant (1986) \\
\hline Acropora palmata & $\mathrm{H}$ & S & Aug & Szmant-Froelich (1984), Szmant (1986) \\
\hline \multicolumn{5}{|l|}{ AGARICIDAE } \\
\hline Agaricia agaricites & $\mathrm{H}$ & B & $\mathrm{sp}$ & Duerden (1902), Van Moorsel (1983) \\
\hline Agaricia crassa & $\mathrm{x}$ & B & $\mathrm{sp}$ & Vaughan $(1910)$ \\
\hline Agaricia fragilis & $\mathrm{x}$ & B & sr & Mavor (1915) \\
\hline Agaricia humilis & $\mathrm{H}$ & B & $\mathrm{yr}$ & Van Moorsel (1983) \\
\hline \multicolumn{5}{|l|}{ FAVIIDAE } \\
\hline Diploria strigosa & $\mathrm{H}$ & $\mathrm{S}$ & Aug, 7 & Szmant-Froelich (1984) \\
\hline \multirow[t]{3}{*}{ Favia fragum } & $\mathrm{H}$ & B & yr & Duerden (1902), Vaughan (1910) \\
\hline & & & $y r, 3-5$ & Szmant-Froelich (1984), Szmant (1986) \\
\hline & & & & Szmant-Froelich et al. (1985) \\
\hline \multirow[t]{2}{*}{ Manicina areolata } & $\mathrm{H}$ & B & $\mathrm{sp}$ & Wilson (1888) in Fadlallah (1983) \\
\hline & & & yr & Duerden (1902) \\
\hline Montastrea annularis & $\mathrm{H}$ & S & Aug, $7 /$ Sep, 7 & Szmant-Froelich (1984), Szmant (1986) \\
\hline Montastrea cavernosa & $G$ & S & Aug & Szmant-Froelich (1984), Szmant (1986) \\
\hline \multicolumn{5}{|l|}{ MEANDRINIDAE } \\
\hline Meandrina ( $=$ Meandra) areolata & $\mathrm{x}$ & B & Jul/Aug, 3-8 & Boschma (1929), Yonge (1935) in Fadlallah (1983) \\
\hline \multicolumn{5}{|l|}{ MUSSIDAE } \\
\hline Isophyllia sp. & $\mathrm{G} ?$ & B & $\mathrm{sp}$ & Duerden (1902) \\
\hline Mycetophyllia ferox & $\mathrm{H}$ & B & Feb-Mar & Szmant-Froelich (1984), Szmant (1986) \\
\hline \multicolumn{5}{|l|}{ PORITIDAE } \\
\hline \multirow[t]{3}{*}{ Porites astreoides } & $\mathrm{H}$ & $\mathrm{B}$ & May-Jun & Vaughan (1910), Szmant-Froelich (1984) \\
\hline & & & Jan-Sep & Szmant (1986) \\
\hline & (or female $o$ & & $y r, 6-8$ & Chornesky \& Peters (1987) \\
\hline Porites porites & $\begin{array}{l}\text { G? } \\
\text { (some herm }\end{array}$ & B & Nov-Feb & Tomascik \& Sander (1987) \\
\hline \multicolumn{5}{|l|}{ SIDERASTREIDAE } \\
\hline \multirow[t]{2}{*}{ Siderastrea radians } & $\mathrm{H}$ & B & yr & Duerden (1902) \\
\hline & $\mathrm{G}$ & $\mathrm{B}$ & yг & Szmant-Froelich (1984), Szmant (1986) \\
\hline Siderastrea siderea & $\mathrm{G}$ & $\mathrm{S}$ & Jul-Sep & Szmant-Froelich (1984), Szmant (1986) \\
\hline \multicolumn{5}{|l|}{ TROCHOSMILIIDAE } \\
\hline \multirow[t]{2}{*}{ Dendrogyra cylindrus } & G & S & Aug & Szmant-Froelich (1984), Szmant (1986) \\
\hline & $6 \mathrm{G}: 10 \mathrm{H}: 3 \mathrm{x}$ & $12 \mathrm{~B}: 7 \mathrm{~S}$ & & \\
\hline \multicolumn{5}{|l|}{ Pacific } \\
\hline \multicolumn{5}{|l|}{ Great Barrier Reef } \\
\hline \multicolumn{5}{|l|}{$\overline{\text { ACROPORIDAE }}$} \\
\hline Acropora aculeus & $\mathrm{H}$ & $\mathrm{S}$ & Nov, 6 & Babcock et al. (1986) \\
\hline Acropora aspera & $\mathrm{H}$ & $\mathrm{S}$ & seasonal & Bothwell (1981) \\
\hline Acropora austera & $\mathrm{H}$ & $\mathrm{S}$ & Nov, 6 & Babcock et al. (1986) \\
\hline Acropora cerealis & $\mathrm{H}$ & $\mathrm{S}$ & Nov, 6 & Babcock et al. (1986) \\
\hline Acropora cuneata & $\mathrm{H}$ & $\mathrm{B}$ & $\mathrm{sp}-\mathrm{sr}$ & Bothwell (1981) \\
\hline Acropora cytherea & $\mathrm{H}$ & $\mathrm{S}$ & Oct/Nov, 6 & Willis et al. (1985), Babcock et al. (1986) \\
\hline \multirow[t]{2}{*}{ Acropora digitifera } & $\mathrm{H}$ & $\mathrm{S}$ & $\mathrm{sp}-\mathrm{sr}$ & Bothwell (1981) \\
\hline & & & Oct, 6 & Willis et al. (1985) \\
\hline Acropora divaricata & $\mathrm{H}$ & $\mathrm{s}$ & Oct, 6 & Willis et al. (1985) \\
\hline Acropora elseyi & $\mathrm{H}$ & $\mathrm{S}$ & Nov, 6/Dec, 1 & Babcock et al. (1986) \\
\hline & & & Oct, 1/Nov, 6 & Willis et al. (1985) \\
\hline Acropora florida & $\mathrm{H}$ & $\mathrm{S}$ & Nov, 6 & $\begin{array}{l}\text { Willis et al. (1985), Wallace (1985b), } \\
\text { Babcock et al. (1986) }\end{array}$ \\
\hline Acropora formosa & $\mathrm{H}$ & S & Nov, 5-6 & Babcock et al. (1986) \\
\hline & & & Oct, $6 /$ Nov, 6 & Willis et al. (1985) \\
\hline Acropora gemmifera & $\mathrm{H}$ & S & Nov, 6 & Willis et al. (1985), Babcock et al. (1986) \\
\hline Acropora grandis & $\mathrm{H}$ & s & Nov, 6 & Willis et al. (1985), Babcock et al. (1986) \\
\hline
\end{tabular}


Table 1 (continued)

\begin{tabular}{|c|c|c|c|c|}
\hline Species & Sex & Mode & Timing & Source \\
\hline \multicolumn{5}{|l|}{ Pacific } \\
\hline \multicolumn{5}{|l|}{ Great Barrier Reef } \\
\hline \multicolumn{5}{|l|}{ ACROPORIDAE } \\
\hline Acropora granulosa & $\mathrm{H}$ & $\mathrm{s}$ & Feb/Mar & Wallace (1985b) \\
\hline Acropora horrida & $\mathrm{H}$ & $\mathrm{S}$ & sr? & Wallace (1985b) \\
\hline \multirow[t]{3}{*}{ Acropora humilis } & $\mathrm{H}$ & $\mathrm{S}$ & $\mathrm{sp}-\mathrm{sr}$ & Bothwell (1981) \\
\hline & & & Oct, 6 & Willis et al. (1985) \\
\hline & & & Nov, $5-7$ & Babcock et al. (1986) \\
\hline \multirow[t]{3}{*}{ Acropora hyacinthus } & $\mathrm{H}$ & $\mathrm{s}$ & $\mathrm{sp}-\mathrm{sr}$ & Bothwell (1981) \\
\hline & & & Oct/Nov, 6 & Willis et al. (1985), Wallace (1985b) \\
\hline & & & Nov, $5-6$ & Babcock et al. (1986) \\
\hline \multirow[t]{2}{*}{ Acropora latistella } & $\mathrm{H}$ & $\mathrm{S}$ & Sep, $6 /$ Oct, 6 & Willis et al. (1985) \\
\hline & & & Nov, 1 & Babcock et al. (1986) \\
\hline \multirow[t]{2}{*}{ Acropora longicyathus } & $H$ & $\mathrm{~S}$ & Nov, 5 & Willis et al. (1985), Wallace (1985b) \\
\hline & & & & Babcock et al. (1986) \\
\hline \multirow[t]{2}{*}{ Acropora loripes } & $\mathrm{H}$ & $\mathrm{S}$ & Nov/Dec & Wallace (1985b) \\
\hline & & & Nov, 6 & Babcock et al. (1986) \\
\hline Acropora lutkeni & $\mathrm{H}$ & $\mathrm{S}$ & Nov, 6 & Babcock at al. (1986) \\
\hline \multirow[t]{2}{*}{ Acropora micropthalma } & $\mathrm{H}$ & $\mathrm{S}$ & Oct, 6 & Willis et al. (1985) \\
\hline & & & Nov, 5 & Babcock et al. (1986) \\
\hline \multirow[t]{3}{*}{ Acropora millepora } & $\mathrm{H}$ & $\mathrm{S}$ & $\mathrm{sp}-\mathrm{sr}$ & Bothwell (1981) \\
\hline & & & Oct, $6 /$ Nov, $5-6$ & Willis et al. (1985) \\
\hline & & & Nov, $5-7$ & Babcock et al. (1986) \\
\hline Acropora nasuta & $\mathrm{H}$ & $\mathrm{S}$ & Nov, $6-7$ & Willis et al.(1985), Babcock et al. (1986) \\
\hline \multirow[t]{3}{*}{ Acropora nobilis } & $\mathrm{H}$ & $\mathrm{S}$ & Oct, 6 & Willis et al. (1985) \\
\hline & & & Oct/Nov & Wallace (1985b) \\
\hline & & & Nov, 5-6 & Babcock et al. (1986) \\
\hline Acropora palifera & $\mathrm{H}$ & $\mathrm{B}$ & $\mathrm{x}$ & Bothwell (1981) \\
\hline \multirow[t]{3}{*}{ Acropora pulchra } & $\mathrm{H}$ & $\mathrm{S}$ & $\mathrm{sp}-\mathrm{sr}$ & Bothwell (1981) \\
\hline & & & Oct, $6-7$ & Willis et al. (1985) \\
\hline & & & Nov, 5 & Babcock et al. (1986) \\
\hline \multirow[t]{2}{*}{ Acropora robusta } & $\mathrm{H}$ & $\mathrm{S}$ & $s p-s r$ & Bothwell (1981) \\
\hline & & & Nov, 6 & Babcock et al. (1986) \\
\hline Acropora samoensis & $\mathrm{H}$ & $\mathrm{S}$ & Nov, 6 & Willis et al. (1985) \\
\hline \multirow[t]{3}{*}{ Acropora samentosa } & $\mathrm{H}$ & $\mathrm{S}$ & Nov, 6 & Willis et al. (1985) \\
\hline & & & Feb/Aug/Nov? & Wallace (1985b) \\
\hline & & & Nov, 7 & Babcock et al. (1986) \\
\hline Acropora secale & $\mathrm{H}$ & $\mathrm{S}$ & Nov, $6-7$ & Babcock et al. (1986) \\
\hline Acropora selago & $\mathrm{H}$ & $\mathrm{S}$ & Nov, 6 & Willis et al. (1985)، Babcock et al. (1986) \\
\hline Acropora solitariensis & $\mathrm{H}$ & $\mathrm{S}$ & Oct, 6 & Willis et al. (1985) \\
\hline \multirow[t]{2}{*}{ Acropora tenuis } & $\mathrm{H}$ & $\mathrm{S}$ & Oct, $6-7 /$ Nov, 6 & Willis et al. (1985) \\
\hline & & & Nov, 5-6 & Babcock et al. (1986) \\
\hline Acropora valenciennesi & $\mathrm{H}$ & $\mathrm{S}$ & Nov, 6 & Willis et al. (1985) \\
\hline Acropora valida & $\mathrm{H}$ & $\mathrm{S}$ & Oct, $6-7 /$ Nov, 6 & Willis et al. (1985). Wallace (1985b) \\
\hline & & & Nov, 5-6 & Babcock et al. (1986) \\
\hline Acropora cf. variabilis & $\mathrm{H}$ & $\mathrm{S}$ & $\mathrm{sp}-\mathrm{sr}$ & Bothwell (1981) \\
\hline Acropora cf. vaughani & $\mathrm{H}$ & $\mathrm{S}$ & Nov, 6 & Willis et al. (1985) \\
\hline Acropora yongei & $\mathrm{H}$ & $\mathrm{S}$ & Nov, 6 & Babcock et al. (1986) \\
\hline Astreopora microphthalma & $\mathrm{H}$ & $\mathrm{S}$ & Nov, 6 & Babcock et al. (1986) \\
\hline Montipora aequituberculata & $\mathrm{H}$ & $\mathrm{S}$ & Oct, 6 & Willis et al. (1985) \\
\hline Montipora digitata & $\mathrm{H}$ & $\mathrm{S}$ & Oct, 5/Nov, 5 & Willıs et al. (1985) \\
\hline & & & Nov, 5 & Babcock et al. (1986), Heyward \& Collins (1985) \\
\hline Montipora foliosa & $\mathrm{H}$ & $\mathrm{S}$ & Nov, 5 & Babcock et al. (1986) \\
\hline Montipora hispida & $\mathrm{H}$ & $\mathrm{S}$ & Oct/Nov, 6 & Willis et al. (1985) \\
\hline & & & Nov, 6 & Babcock et al. (1986) \\
\hline Montipora informis & $\mathrm{H}$ & $\mathrm{S}$ & Oct, 5 & Willis et al. (1985) \\
\hline Montipora monasteriata & $\mathrm{H}$ & $\mathrm{S}$ & Nov, 5 & Babcock et al. (1986) \\
\hline Montipora spumosa & $\mathrm{H}$ & $\mathrm{S}$ & Nov, 5 & Babcock et al. (1986) \\
\hline Montipora tuberculosa & $\mathrm{H}$ & $\mathrm{S}$ & Nov, 6 & Babcock et al. (1986) \\
\hline Montipora turgescens & $\mathrm{H}$ & $\mathrm{S}$ & Nov, 6 & Babcock et al. (1986) \\
\hline AGARICIIDAE & & & & \\
\hline Pachyseris rugosa & $G$ & $S$ & Nov, 5-6 & Willıs et al. (1985) \\
\hline & & & Nov, 6 & Babcock et al. (1986) \\
\hline
\end{tabular}


Table 1 (continued)

\begin{tabular}{|c|c|c|c|c|}
\hline Species & Sex & Mode & Timing & Source \\
\hline \multicolumn{5}{|l|}{ Pacific } \\
\hline \multicolumn{5}{|l|}{ Great Barrier Reef } \\
\hline \multicolumn{5}{|l|}{ AGARICIIDAE } \\
\hline \multirow[t]{2}{*}{ Pachyseris speciosa } & G & $S$ & Oct, $6-7 /$ Nov, $5-6$ & Willis et al. (1985) \\
\hline & & & Nov, $6-7$ & Babcock et al. (1986) \\
\hline Pavona cactus & $G(?)$ & S(sperm) & $\mathrm{x}$ & Marshall \& Stephenson (1933) \\
\hline \multicolumn{5}{|l|}{ CARYOPHYLLIDAE } \\
\hline Catalaphylia jardineri & $\mathrm{G}$ & $\mathrm{S}$ & Oct, 6 & Willis et al. (1985) \\
\hline Euphyllia ancora & $G$ & $\mathrm{~S}$ & Oct, 6 & Willis et al. (1985) \\
\hline \multirow[t]{2}{*}{ Euphyllia divisa } & $G$ & $\mathrm{~S}$ & Oct, 6 & Willis et al. (1985) \\
\hline & & & Nov, 6 & Babcock et al. (1986) \\
\hline \multirow[t]{2}{*}{ Physogyra lichtensteini } & $G$ & $\mathrm{~S}$ & Nov, 6 & Willis et al. (1985) \\
\hline & & & & Babcock et al. (1986) \\
\hline \multicolumn{5}{|l|}{ DENDROPHYLLIDAE } \\
\hline Dendrophyllia sp. & $G$ & $\mathrm{~B}$ & Dec, 1 & Babcock et al. (1986) \\
\hline Heteropsammia cochlea & $G$ & $\mathrm{~S}$ & Jan-Jun & Fisk (1981) in Harriott (1983a) \\
\hline Heteropsammia aequicostatus & G & $\mathrm{S}$ & Apr-Jun & Fisk (1981) in Harriott (1983a) \\
\hline Tubastrea faulkneri & G & $\mathrm{B}$ & Nov, $6-7$ & Babcock et al. (1986) \\
\hline Turbinaria frondens & G & $\mathrm{S}$ & Oct, 6 & Willis et al. (1985) \\
\hline Turbinaria reniformis & G & $\mathrm{S}$ & Nov, 7 & Willis et al. (1985) \\
\hline \multicolumn{5}{|l|}{ FAVIIDAE } \\
\hline Australogyra zelli & $\mathrm{H}$ & $\mathrm{S}$ & Nov, 6 & Willis et al. (1985), Babock et al. (1986) \\
\hline Barbattoia amicorum & $\mathrm{H}$ & $\mathrm{s}$ & Nov, $5-7$ & Babcock et al. (1986) \\
\hline Caulastrea furcata & $\mathrm{H}$ & $\mathrm{S}$ & Nov, 5 & Willis et al. (1985), Babcock et al. (1986) \\
\hline \multirow[t]{2}{*}{ Cyphastrea chalcidium } & $\mathrm{H}$ & $\mathrm{S}$ & Oct, 6 & Willis et al. (1985) \\
\hline & & & Nov, 6 & Babcock et al. (1986) \\
\hline \multirow[t]{2}{*}{ Cyphastrea micropthalma } & $\mathrm{H}$ & $\mathrm{S}$ & Oct, 6 & Willis et al. (1985) \\
\hline & & & Nov, 6 & Babcock et al. (1986) \\
\hline Cyphastrea serailia & $\mathrm{H}$ & $\mathrm{S}$ & Oct, 6 & Willis et al. (1985) \\
\hline Diploastrea heliopora & G & $\mathrm{x}$ & $\mathrm{x}$ & Harrison (1985) \\
\hline Echinopora gemmacea & $\mathrm{H}$ & $S$ & Nov, 6 & Babcock et al. (1986) \\
\hline Echinopora horrida & $\mathrm{H}$ & $\mathrm{S}$ & Nov, 6 & Babcock et al. (1986) \\
\hline \multirow[t]{2}{*}{ Echinopora lamellosa } & $\mathrm{H}$ & $\mathrm{S}$ & Oct, $6 /$ Nov, 5 & Willis et al. (1985) \\
\hline & & & Nov, 6 & Babcock et al. (1986) \\
\hline \multirow{2}{*}{ Favia favus } & $\mathrm{H}$ & $\mathrm{S}$ & Nov/Dec & Harriott (1983a) \\
\hline & & & Nov, 6 & Babcock et al (1986) \\
\hline Favia lizardensis & $\mathrm{H}$ & $\mathrm{S}$ & Nov, 6 & Babcock et al. (1986) \\
\hline \multirow[t]{2}{*}{ Favia mathaii } & $\mathrm{H}$ & $\mathrm{S}$ & Nov, 5-6 & Willis et al. (1985) \\
\hline & & & & Babcock et al. (1986) \\
\hline Favia pallida (as $F$ doreyensis) & $\mathrm{H}$ & $\mathrm{S}$ & Dec & Marshall \& Stephenson (1933) \\
\hline \multirow[t]{2}{*}{ Favia pallida } & & & Oct/Nov, 6 & Willis et al. (1985) \\
\hline & & & Nov, 6 & Babcock et al. (1986) \\
\hline Favia rotumana & $\mathrm{H}$ & $\mathrm{s}$ & Nov. 6 & Willis et al. (1985) \\
\hline Favia stelligera & $\mathrm{H}$ & $\mathrm{S}$ & Nov, 6 & Babcock et al. (1986) \\
\hline Favia veroni & $\mathrm{H}$ & $\mathrm{S}$ & Nov, 5-7 & Babcock et al. (1986) \\
\hline Favites abdita & $\mathrm{H}$ & $S$ & Nov, 6 & Kojis \& Quinn (1982), Babcock et al. (1986) \\
\hline & & & Nov, 5-6 & Willis et al. (1985) \\
\hline Favites bennettae & $\mathrm{H}$ & $\mathrm{S}$ & Nov, $5-7$ & Babcock et al. (1986) \\
\hline Favites chinensis & $\mathrm{H}$ & $\mathrm{S}$ & Nov, 6 & Willis et al. (1985) \\
\hline Favites complanata & $\mathrm{H}$ & $\mathrm{S}$ & Nov, 6 & Willis et al. (1985), Babcock et al. (1986) \\
\hline Favites flexuosa & $\mathrm{H}$ & $\mathrm{S}$ & Nov, 6 & Willis et al. (1985), Babcock et al. (1986) \\
\hline Favites halicora & $\mathrm{H}$ & $\mathrm{S}$ & Nov, 6 & Willis et al. (1985), Babcock et al. (1986) \\
\hline Favites pentagona & $\mathrm{H}$ & $\mathrm{S}$ & Oct, 6 & Willis et al. (1985) \\
\hline & & & Nov, 6 & Babcock et al. (1986) \\
\hline Favites russelli & $\mathrm{H}$ & $\mathrm{S}$ & Nov, 6 & Babcock et al. (1986) \\
\hline Goniastrea aspera & $\mathrm{H}$ & $\mathrm{S}$ & Oct, $5-7$ & Babcock $(1984)$ \\
\hline & & & Oct, $6-7 /$ Nov, 6 & Willis et al. (1985) \\
\hline & & & Nov, 5-6 & Babcock et al. (1986) \\
\hline Goniastrea edwardsi & $\mathrm{H}$ & $\mathrm{S}$ & Nov, 6 & Babcock et al. (1986) \\
\hline $\begin{array}{l}\text { Coniastrea favulus } \\
\text { (as } G \text {. australensis) }\end{array}$ & $\mathrm{H}$ & $\mathrm{S}$ & Oct/Nov, 5-6 & Kojis \& Quinn (1981a), Kojis \& Quinn (1982) \\
\hline Goniastrea favulus & & & Oct, 6/Nov, 5-6 & Willis et al. (1985) \\
\hline & & & Nov, $5-6$ & Babcock et al. (1986) \\
\hline
\end{tabular}


Table 1 (continued)

\begin{tabular}{|c|c|c|c|c|}
\hline Species & Sex & Mode & Timing & Source \\
\hline \multicolumn{5}{|l|}{ Pacific } \\
\hline \multicolumn{5}{|l|}{ Great Barrier Reef } \\
\hline \multicolumn{5}{|l|}{ FAVIIDAE } \\
\hline \multirow[t]{2}{*}{ Goniastrea palauensis } & $\mathrm{H}$ & $\mathrm{s}$ & Oct, 6 & Willis et al. (1985) \\
\hline & & & Nov, 6 & Babcock et al. (1986) \\
\hline \multirow[t]{2}{*}{ Goniastrea pectinata } & $\mathrm{H}$ & $\mathrm{S}$ & Nov, 6 & Willis et al. (1985) \\
\hline & & & & Babcock et al. (1986) \\
\hline Goniastrea retiformis & $\mathrm{H}$ & $\mathrm{s}$ & Nov, 6 & Willis et al. (1985), Babcock et al. (1986) \\
\hline \multirow[t]{2}{*}{ Leptoria phrygia } & $\mathrm{H}$ & $\mathrm{S}$ & Dec & Kojis \& Quinn (1982) \\
\hline & & & Nov, 6 & Willis et al. (1985), Babcock et al. (1986) \\
\hline Montastrea curta & $\mathrm{H}$ & $\mathrm{S}$ & Nov, 6 & Willis et al. (1985), Babcock et al. (1986) \\
\hline Montastrea magnistellata & $\mathrm{H}$ & $\mathrm{S}$ & Nov, 6 & Willis et al. (1985), Babcock et al. (1986) \\
\hline \multirow[t]{2}{*}{ Montastrea valenciennesi } & $\mathrm{H}$ & $\mathrm{S}$ & Oct, 6 & Willis et al. (1985) \\
\hline & & & Nov, 6 & Babcock et al. (1986) \\
\hline \multirow[t]{2}{*}{ Moseleya latistellata } & $\mathrm{H}$ & $\mathrm{s}$ & Oct, $6-8$ & Willis et al. (1985) \\
\hline & & & Nov, 6 & Babcock et al. (1986) \\
\hline Oulophyllia crispa & $\mathrm{H}$ & $\mathrm{S}$ & Nov, 6 & Willis et al. (1985), Babcock et al. (1986) \\
\hline \multirow[t]{2}{*}{ Platygyra daedalea } & $\mathrm{H}$ & $\mathrm{S}$ & Oct, 6-7 & Willis et al. (1985), Babcock et al. (1986) \\
\hline & & & Nov, 6-7 & \\
\hline Platygyra lamellina & $\mathrm{H}$ & $\mathrm{S}$ & Nov, $6-7$ & Babcock et al. (1986) \\
\hline Platygyra pini & $\mathrm{H}$ & $\mathrm{S}$ & Nov, 6 & Babcock et al. (1986) \\
\hline \multirow[t]{3}{*}{ Platygyra sinensis } & $\mathrm{x}$ & $\mathrm{S}$ & $\mathrm{x}-1$ & Babcock $(1984)$ \\
\hline & & & Oct, $6 /$ Nov, 6 & Willis et al. (1985) \\
\hline & $\mathrm{H}$ & & Nov, $6-7$ & Babcock et al. (1986) \\
\hline \multicolumn{5}{|l|}{ FUNGIIDAE } \\
\hline Fungia concinna & $\mathrm{G}$ & $\mathrm{S}$ & Nov, $7-8$ & Willis et al. (1985) \\
\hline \multirow{2}{*}{ Fungia fungites } & G & $\mathrm{S}$ & Oct, 6 & Willis et al. (1985) \\
\hline & & & Nov, 6 & Babcock et al. (1986) \\
\hline Fungia paumotensis & G & $\mathrm{S}$ & Nov, 6 & Willis et al. (1985) \\
\hline Heliofungia actiniformis & G & $\mathrm{S}$ & Oct, $5 /$ Nov, 5 & Willis et al. (1985) \\
\hline Polyphyllia talpina & $G$ & $\mathrm{~S}$ & Oct, $6 /$ Nov, 6 & Willis et al. (1985) \\
\hline Sandolitha robusta & $\mathrm{G}$ & $S$ & Nov, 6 & Babcock et al. (1986) \\
\hline \multicolumn{5}{|l|}{ MERULINIDAE } \\
\hline Clavarina triangularis & $\mathrm{H}$ & $\mathrm{S}$ & Nov, 6 & Babcock et al. (1986) \\
\hline Merulina ampliata & $\mathrm{H}$ & $\mathrm{S}$ & Nov, 6 & Babcock et al. (1986) \\
\hline Scapophyllia cylindrica & $\mathrm{H}$ & $\mathrm{S}$ & Nov, 6 & Willis et al. (1985) \\
\hline \multicolumn{5}{|l|}{ MUSSIDAE } \\
\hline Acanthastrea echinata & $\mathrm{H}$ & $\mathrm{S}$ & Nov, 5-? & Willis et al. (1985), Babcock et al. (1986) \\
\hline \multirow{2}{*}{ Lobophyllia corymbosa } & $\mathrm{H}$ & s & Nov/Dec & Harriott $(1983 a)$ \\
\hline & & & Nov, 6 & Willis et al. (1985), Babcock et al. (1986) \\
\hline Lobophyllia hemprichii & $\mathrm{H}$ & $\mathrm{S}$ & Oct/Nov, $6-7$ & Willis et al. (1985) \\
\hline & & & Nov, 6 & Babcock et al. (1986) \\
\hline Scolymia vitiensis & $\mathrm{H}$ & $\mathrm{S}$ & Nov, 6 & Willis et al. (1985) \\
\hline Symphyllia radians & $\mathrm{H}$ & $\mathrm{S}$ & Nov, 6 & Babcock et al. (1986) \\
\hline Symphyllia recta & $\mathrm{H}$ & $\mathrm{S}$ & $\mathrm{sp}-\mathrm{sr}$ & Marshall \& Stephenson (1933) \\
\hline & & & Nov, 6 & Willis et al. (1985) \\
\hline & & & & Babcock et al. (1986) \\
\hline OCULINIDAE & & & & \\
\hline Galaxea astreata & $\mathrm{H}$ & $\mathrm{S}$ & Oct/Nov, 6 & Willis et al. (1985) \\
\hline & & & Nov, 6 & Babcock et al. (1986) \\
\hline Galaxea fascicularis & $\mathrm{H}$ & $\mathrm{S}$ & Oct/Nov, 6 & Willis et al. (1985) \\
\hline & & & Nov, 6 & Babcock et al. (1986) \\
\hline PECTINIDAE & & & & \\
\hline Echinophyllia aspera & $\mathrm{H}$ & $\mathrm{s}$ & Oct/Nov, 7-8 & Willis et al. (1985) \\
\hline & & & Nov, 6 & Babcock et al. (1986) \\
\hline Echinophyllia orpheensis & $\mathrm{H}$ & $\mathrm{S}$ & Nov, 6 & Babcock et al. (1986) \\
\hline Myoedium elephantotus & $\mathrm{H}$ & $\mathrm{S}$ & Oct, $6-7$ Nov. 6 & Willis et al. (1985) \\
\hline & & & Nov, 6 & Babcock et al. (1986) \\
\hline Oxypora glabra & $\mathrm{H}$ & $\mathrm{S}$ & Nov, $5-6$ & Babcock et al. (1986) \\
\hline Oxypora lacera & $\mathrm{H}$ & $\mathrm{S}$ & Oct, $6 /$ Nov, 6 & Willis et al. (1985) \\
\hline & & & Nov, 6 & Babcock et al. (1986) \\
\hline Pectinia alcicornis & $\mathrm{H}$ & $\mathrm{S}$ & Nov, 6 & Willis et al. (1985), Babcock et al. (1986) \\
\hline
\end{tabular}


Table 1 (continued)

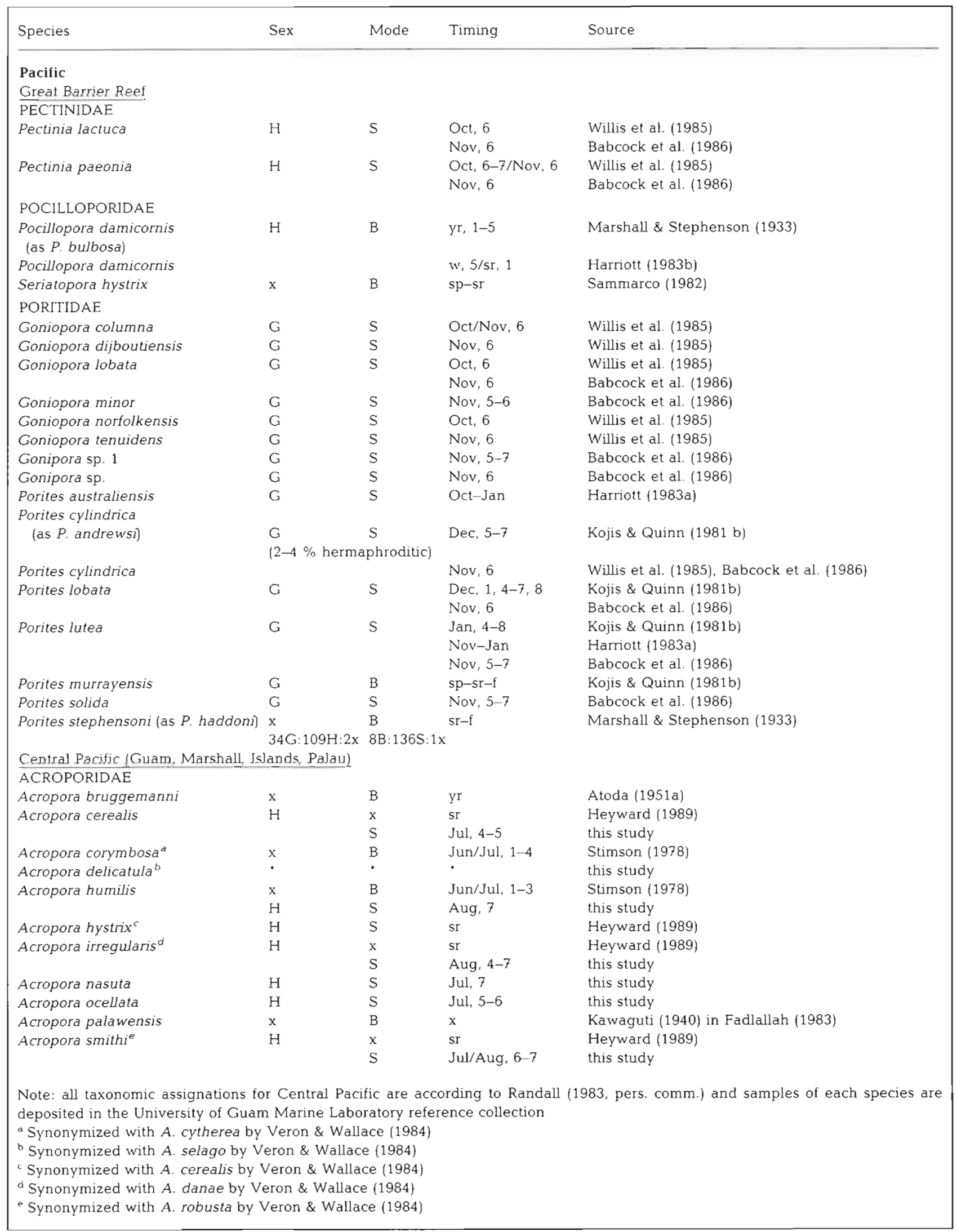


Table 1 (continued)

\begin{tabular}{|c|c|c|c|c|}
\hline Species & Sex & Mode & Timing & Source \\
\hline \multicolumn{5}{|c|}{ Pacific } \\
\hline \multicolumn{5}{|c|}{ Central Pacific (Guam, Marshall, Islands, Palau) } \\
\hline \multicolumn{5}{|c|}{ ACROPORIDAE } \\
\hline Acropora squarrosa ${ }^{\prime}$ & $\mathrm{H}$ & $\mathrm{S}$ & Jul, 2-3 & this study \\
\hline Acropora striata & $\mathrm{x}$ & B & $\begin{array}{l}\text { Jun/Jul, 3-4 } \\
\text { Jan, } 1-8\end{array}$ & Stimson (1978) \\
\hline Acropora surculosa ${ }^{g}$ & $\mathrm{H}$ & S & Jul, $7-8$ & this study \\
\hline Acropora tenuis & $\mathrm{H}$ & $\mathrm{S}$ & Jul, 7-8 & this study \\
\hline Acropora valida & $\mathrm{H}$ & $\mathrm{x}$ & sr & Heyward (1989) \\
\hline Acropora variabilis ${ }^{h}$ & $\mathrm{H}$ & $\begin{array}{l}S \\
S\end{array}$ & $\begin{array}{l}\text { Jul, } 6-7 \\
\text { sr }\end{array}$ & $\begin{array}{l}\text { this study } \\
\text { Heyward (1989) }\end{array}$ \\
\hline Astreopora randalli & $\mathrm{H}$ & $\mathrm{S}$ & Jul, 8/Aug, 1 & this study \\
\hline Montipora foveolata & $\mathrm{H}$ & $\mathrm{S}$ & Jul, 2-3 & this study \\
\hline Montipora verrucosa & $\mathrm{H}$ & S & Jun, $7-8$ & this study \\
\hline \multicolumn{5}{|l|}{ CARYOPHYLLIDAE } \\
\hline Euphyllia glabrescens & $\mathrm{x}$ & B & $\mathrm{x}$ & Kawaguti (1941) \\
\hline \multicolumn{5}{|l|}{ DENDROPHYLLIDAE } \\
\hline Balanophyllia sp. & $\mathrm{x}$ & $\mathrm{B}$ & $\mathrm{yr}$ & Abe (1937) \\
\hline \multicolumn{5}{|l|}{ FAVIIDAE } \\
\hline \multirow[t]{2}{*}{ Favia mathaii } & $\mathrm{H}$ & $x$ & sr & Heyward (1989) \\
\hline & & $\mathrm{S}$ & Jun/Jul, 6-8 & this study \\
\hline Favia stelligera & $\mathrm{H}$ & $\mathrm{S}$ & Jun/Jul, 5-7 & this study \\
\hline Favites abdita & $\mathrm{H}$ & $\mathrm{s}$ & sr & Heyward (1989) \\
\hline Favites flexuosa & $\mathrm{H}$ & $\mathrm{s}$ & sr & Heyward (1989) \\
\hline Goniastrea aspera & $\mathrm{x}$ & B & Oct/Nov, 1 & Abe (1937) \\
\hline \multirow[t]{2}{*}{ Goniastrea edwardsi } & $\mathrm{H}$ & $S$ & sr & Heyward (1989) \\
\hline & & & Jun/Jul, 7-8 & this study \\
\hline Goniastrea retiformis & $\mathrm{H}$ & $\mathrm{s}$ & Jul, $1-2 / 7-8$ & this study \\
\hline \multirow{2}{*}{ Leptoria phrygia } & $\mathrm{H}$ & $\mathrm{x}$ & $\mathrm{sr}$ & Heyward (1989) \\
\hline & & s & Jul, $7-8$ & this study \\
\hline Montastrea curta & $\mathrm{H}$ & $\mathrm{s}$ & Aug, $6-7$ & this study \\
\hline Platygyra daedalea & $\mathrm{H}$ & $\mathrm{s}$ & Jul, $7-8$ & this study \\
\hline Platygyra pini & $\mathrm{H}$ & S & $\mathrm{sr}$ & Heyward (1989) \\
\hline \multicolumn{5}{|l|}{ FUNGIIDAE } \\
\hline Fungia fungites & $G$ & s & sr & Heyward (1989) \\
\hline Heliofungia actiniformis & $\mathrm{H}$ ? & B & Sep-Apr. 1 & Abe (1937) \\
\hline \multicolumn{5}{|l|}{ OCULINIDAE } \\
\hline Acrhelia horrescens & $\mathrm{x}$ & B & $\mathrm{yr}$ & Kawaguti (1941) \\
\hline \multirow{2}{*}{$\begin{array}{l}\text { Galaxea fascicularis } \\
\text { (as } G \text {. aspera) }\end{array}$} & $\mathrm{H}$ & S & Jul/Aug, 1-3 & this study \\
\hline & $\mathrm{x}$ & B & yr & Atoda (1951b) \\
\hline \multicolumn{5}{|l|}{ POCILLOPORIDAE } \\
\hline \multirow{4}{*}{$\begin{array}{l}\text { Pocillopora damicomis } \\
\text { (as P. caespitosa) }\end{array}$} & $\mathrm{H}$ & B & $\mathrm{yr}$ & Hada (1932), Kawaguti (1941) \\
\hline & & & yr, $1-3$ & Atoda (1947a) \\
\hline & & & Jun/Jul/Jan, 7-3 & Stımson (1978) \\
\hline & & & yr, 2-3 & Richmond \& Jokiel (1984) \\
\hline $\begin{array}{c}\text { Pocillopora elegans } \\
(P . \text { meandrina? })\end{array}$ & $\mathrm{x}$ & B & $\operatorname{Jan}, 3-4$ & Stimson $(1978)$ \\
\hline Pocillopora verrucosa & $\mathrm{x}$ & B & $\begin{array}{l}\text { Jun/Jul, 1-3 } \\
\text { Jan, 1-4 }\end{array}$ & Strmson $(1978)$ \\
\hline Stylophora pistillata & $\mathrm{x}$ & B & yr, $5-7$ & Atoda $(1947 b)$ \\
\hline Seriatopora hystrix & $\mathrm{x}$ & B & $s r / w, 1-5$ & Kawaguti (1941), Atoda (1951c), Stimson (1978) \\
\hline \multicolumn{5}{|l|}{ PORITIDAE } \\
\hline Goniopora fruticosa & $\cdot$ & $\cdot$ & $\cdot$ & this study \\
\hline Porites cylindrica & $\cdot$ & $\cdot$ & $\cdot$ & this study \\
\hline \multicolumn{5}{|c|}{$\begin{array}{l}\text { Note: all taxonomic assignations for Central Paciftc are accord } \\
\text { deposited in the University of Guam Manne Laboratory referen } \\
\text { f Synonymized with A. loripes by Veron \& Wallace (1984) } \\
\text { g Synonymued with A. hyacinthus by Veron \& Wallace (1984) } \\
\text { h Synonymized with A. valida by Veron \& Wallace (1984) }\end{array}$} \\
\hline
\end{tabular}


Table 1 (continued)

\begin{tabular}{|c|c|c|c|c|}
\hline Species & Sex & Mode & Timing & Source \\
\hline \multicolumn{5}{|c|}{ Pacific } \\
\hline \multicolumn{5}{|c|}{ Central Pacific (Guam, Marshall, Islands, Palau) } \\
\hline \multicolumn{5}{|c|}{ PORITIDAE } \\
\hline Porites lobata & G & $\mathrm{S}$ & Jul, 7-8 & this study \\
\hline Porites lutea & G & $\mathrm{x}$ & sr & Heyward (1989) \\
\hline \multirow[t]{2}{*}{ Porites (Synarea) rus } & G & $\mathrm{x}$ & $\mathrm{x}$ & this study \\
\hline & $4 \mathrm{G}: 28 \mathrm{H}: 3^{\bullet}: 12 \mathrm{x}$ & $14 \mathrm{~B}: 28 \mathrm{~S}: 3^{\bullet}: 2 \mathrm{x}$ & & \\
\hline \multicolumn{5}{|l|}{ Hawaii } \\
\hline \multicolumn{5}{|l|}{ ACROPORIDAE } \\
\hline Acropora cytherea & $\cdot$ & $\cdot$ & $\cdot$ & Grigg et al. (1981) \\
\hline Acropora humilis & . & $\cdot$ & . & Grigg et al. (1981) \\
\hline Acropora valida & $\cdot$ & $\cdot$ & $\cdot$ & Grigg et al. (1981) \\
\hline \multirow[t]{3}{*}{ Montipora dilatata } & $\mathrm{H}$ & S & Jul, $5-6$ & Heyward (1985) \\
\hline & & & Jun/Jul/Aug & Heyward (1985) \\
\hline & & & $\begin{array}{l}\text { Jul Aug, } 5-7 \\
\text { Jul, } 1\end{array}$ & Hunter (1989) \\
\hline Montipora flabellata & $\mathrm{H}$ & $\mathrm{S}$ & sr- $\mathrm{f}$ & Heyward (1985) \\
\hline Montipora studeryi & $\mathrm{H}$ & $\mathrm{S}$ & Jul, 5-6 & Heyward (1985) \\
\hline \multirow{2}{*}{$\begin{array}{l}\text { Montipora verrucosa } \\
\text { (sensu Vaughan 1907) }\end{array}$} & $\mathrm{H}$ & $\mathrm{S}$ & Jul, 1 & Heyward (1985) \\
\hline & & & Jun/Jul, 1 & Hunter (1989) \\
\hline Montipora verrilli & $\mathrm{H}$ & $\mathrm{S}$ & Jul, 5-6 & Heyward (1985) \\
\hline \multicolumn{5}{|l|}{ DENDROPHYLLIDAE } \\
\hline Dendrophyllia manni & $\mathrm{x}$ & $\mathrm{B}$ & $s r-f$ & Edmondson (1929), Edmondson (1946) \\
\hline $\begin{array}{l}\text { Tubastrea coccinea } \\
\text { (as } T \text { aurea) }\end{array}$ & $\mathrm{x}$ & B & $\mathrm{sr}-\mathrm{w}$ & Edmondson (1929), Edmondson (1946) \\
\hline \multicolumn{5}{|l|}{ FAVIIDAE } \\
\hline Cyphastrea ocellina & $\mathrm{x}$ & $\mathrm{B}$ & yr & $\begin{array}{l}\text { Edmondson (1929), Edmondson (1946), Stimson } \\
\text { (1978) }\end{array}$ \\
\hline \multicolumn{5}{|l|}{ FUNGIIDAE } \\
\hline Fungia scutaria & $G$ & $\mathrm{~S}$ & Jul-Sep, 5 & Krupp (1983) \\
\hline \multicolumn{5}{|l|}{ POCILLOPORIDAE } \\
\hline \multirow[t]{3}{*}{ Pocillopora damicornis } & $\mathrm{H}$ & B & yr, 5-1 & Edmondson (1946), Harrigan (1972) \\
\hline & & & Jun/Jul/Aug, 1 & Reed (1971) \\
\hline & & & $y \mathrm{r}, 5$ & Stimson (1978) \\
\hline 'Type Y' & & & yr, $6-8$ & Richmond \& Jokiel (1984) \\
\hline 'Type B' & & & $y r, 3-5$ & \\
\hline \multicolumn{5}{|l|}{ PORITIDAE } \\
\hline Porites compressa & $\mathrm{G}$ & S & Jun-Aug, 5 & Hunter (1988), Hunter \& Hodgson unpubl. \\
\hline Porites evermanni & $G$ & S & Aug-Sep, 5-6 & Hunter \& Hodgson unpubl. \\
\hline Porites lobata & G & $\mathrm{S}$ & Aug, $7-8$ & Hunter \& Hodgson unpubl. \\
\hline \multirow[t]{2}{*}{ Porites brighami } & $\mathrm{x}$ & $\mathrm{B}$ & sr & Hunter \& Hodgson unpubl. \\
\hline & $4 \mathrm{G}: 6 \mathrm{H}: 3^{\bullet}: 4 \mathrm{x}$ & $5 \mathrm{~B}: 9 \mathrm{~S}: 3^{\bullet}$ & & \\
\hline \multicolumn{5}{|l|}{ Okinawa } \\
\hline \multicolumn{5}{|l|}{ ACROPORIDAE } \\
\hline Acropora anthocercis & $\mathrm{H}$ & S & Jun, 5 & Richmond pers. obs. \\
\hline Acropora cytherea & $\mathrm{H}$ & $S$ & Jun, 5 & Heyward et al. (1987), Richmond pers. obs. \\
\hline Acropora digitifera & $\mathrm{H}$ & s & Jun, 5 & Richmond pers. obs. \\
\hline Acropora florida & $\mathrm{H}$ & $\mathrm{S}$ & Jun, $5-8$ & Heyward et al (1987), Richmond pers. obs. \\
\hline Acropora formosa & $\mathrm{H}$ & S & Jun, 5-8 & Heyward et al (1987) \\
\hline Acropora grandis & $\mathrm{H}$ & S & Jun, 5 & Heyward et al. (1987) \\
\hline Acropora hyacynthus & $\mathrm{H}$ & S & Jun, 5 & Richmond pers obs. \\
\hline Acropra latistella & $\mathrm{H}$ & S & Jun, 7 & Heyward et al. (1987) \\
\hline \multirow[t]{2}{*}{ Acropora micropthalma } & $\mathrm{H}$ & S & Jun. 7 & Heyward et al. (1987) \\
\hline & & & Jun, 5 & Richmond pers. obs. \\
\hline Acropora nobilis & $\mathrm{H}$ & S & Jun, 5 & Richmond pers. obs. \\
\hline Acropora tenuis & $\mathrm{H}$ & $\mathrm{S}$ & Jun, 5 & K. Sakai pers. comm. \\
\hline Acropora valida & $\mathrm{H}$ & S & Jun, 5 & K. Sakai pers. comm. \\
\hline Montipora aequituberculata & $\mathrm{H}$ & $S$ & Jun, 5-6 & Heyward et al. (1987) \\
\hline Montipora digitata & $\mathrm{H}$ & S & Jun, 5-6 & Heyward et al. (1987) \\
\hline Montipora effusa & $\mathrm{H}$ & S & Jul, 6/Aug, 6-7 & Heyward et al. (1987) \\
\hline
\end{tabular}


Table 1 (continued)

\begin{tabular}{|c|c|c|c|c|}
\hline Species & Sex & Mode & Timing & Source \\
\hline \multicolumn{5}{|l|}{ Pacific } \\
\hline \multicolumn{5}{|l|}{ Okinawa } \\
\hline \multicolumn{5}{|l|}{$\overline{\text { ACROPORIDAE }}$} \\
\hline Montipora turgescens & $\mathrm{H}$ & $\mathrm{S}$ & Jun, 5 & Heyward et al. (1987) \\
\hline \multicolumn{5}{|l|}{ FAVIIDAE } \\
\hline Favia pallida & $\mathrm{H}$ & $\mathrm{s}$ & Jun, 5 & Heyward et al. (1987) \\
\hline \multirow[t]{2}{*}{ Favites chinensis } & $\mathrm{H}$ & $S$ & Jun, $6-7 / \mathrm{Jul}, 7$ & Heyward et al. (1987) \\
\hline & & & Aug, 7 & Heyward et al. (1987) \\
\hline Goniastrea aspera & $\mathrm{H}$ & $\mathrm{S}$ & Jun/Jul, 5-6 & Heyward et al. (1987) \\
\hline Platygyra pini & $\mathrm{H}$ & $S$ & Jun, 2 & Heyward et al. (1987) \\
\hline Platygyra ryukuensis & $\mathrm{H}$ & S & Jul, 7/Aug, 6-7 & Heyward et al. (1987) \\
\hline \multicolumn{5}{|l|}{ FUNGIIDAE } \\
\hline Fungia sp. & G & S & Jul, 7 & Heyward et al. (1987) \\
\hline \multicolumn{5}{|l|}{ MUSSIDAE } \\
\hline Lobophyllia corymbosa & $\mathrm{H}$ & $S$ & Jun, 6 & Heyward et al. (1987) \\
\hline Symphyllia recta & $\mathrm{H}$ & s & Jun, 5 & Richmond pers obs. \\
\hline \multicolumn{5}{|l|}{ OCULINIDAE } \\
\hline Galaxea fascicularis & $\mathrm{H}$ & $S$ & Jun/Jul/Aug, 6-7 & Heyward et al. (1987) \\
\hline \multicolumn{5}{|l|}{ PORITIDAE } \\
\hline \multirow[t]{2}{*}{ Gonipora queenslandiae } & $\mathrm{G}$ & B & Jul/Aug & Yamazato et al. (1975) \\
\hline & $2 \mathrm{G}: 24 \mathrm{H}$ & $1 \mathrm{~B}: 25 \mathrm{~S}$ & & \\
\hline \multicolumn{5}{|c|}{ Eastern Pacific - work in progress } \\
\hline Pocillopora damicornis & \multicolumn{4}{|c|}{$\begin{array}{l}\text { After } 2 \text { yr, only immature ovaries observed (spring). No complete gametogenesis, spawnung or } \\
\text { planulation observed (Richmond 1985) }\end{array}$} \\
\hline Pocillopora elegans & \multicolumn{4}{|c|}{ Spermaries and ovaries observed near maturity during summer (A. Yedid pers. comm.) } \\
\hline Tubastrea aurea & \multicolumn{4}{|c|}{ Planulated Jun through Nov during both 1984 and 1985 (Richmond unpubl.) } \\
\hline \multicolumn{5}{|l|}{ Red Sea } \\
\hline \multicolumn{5}{|l|}{ ACROPORIDAE } \\
\hline Acropora eurystoma & $\mathrm{H}$ & S & May/Jun, 5 & Shlesinger \& Loya $(1985)$ \\
\hline Acropora hemprichii & $\mathrm{H}$ & $\mathrm{x}$ & $\mathrm{x}$ & Rinkevich \& Loya (1979a) \\
\hline Acropora humilis & $\mathrm{H}$ & S & May/Jun, 7 & Shlesinger \& Loya (1985) \\
\hline Acropora hyacinthus & $\mathrm{H}$ & $\mathrm{S}$ & Jul, 3 & Shlesinger \& Loya (1985) \\
\hline Acropora scandens & $\mathrm{H}$ & $\mathrm{S}$ & Jun/Jul, 5 & Shlesinger \& Loya (1985) \\
\hline Astreopora myriophthalma & $\mathrm{H}$ & $\mathrm{S}$ & Jul/Aug/Sep, 5 & Shlesinger \& Loya (1985) \\
\hline \multicolumn{5}{|l|}{ FAVIIDAE } \\
\hline Favia favus & $\mathrm{H}$ & $S$ & $\begin{array}{l}\text { Jun/Jul, } 6-7 \\
\text { Aug, } 6\end{array}$ & Shlesinger \& Loya (1985) \\
\hline Favites abdita & $\mathrm{H}$ & $s$ & $\mathrm{x}$ & Rinkevich \& Loya (1979a) \\
\hline Goniastrea retiformis & $\mathrm{H}$ & $\mathrm{S}$ & Jul/Aug, 7 & Shlesinger \& Loya (1985) \\
\hline \multirow[t]{2}{*}{ Platygyra lamellina } & $\mathrm{H}$ & $\mathrm{S}$ & Jun/Jul, 1-2 & Rinkevich \& Loya (1979a) \\
\hline & & & Aug, 1 & Shlesinger \& Loya (1985) \\
\hline \multicolumn{5}{|l|}{ OCULINIDAE } \\
\hline Galaxea fascicularis & $\mathrm{H}$ & s & Jul/Aug/Sep, 6-7 & Shlesinger \& Loya (1985) \\
\hline \multicolumn{5}{|l|}{ POCILLOPORIDAE } \\
\hline \multirow[t]{2}{*}{ Pocillopora verrucosa } & $\mathrm{H}$ & S & May, 1 & Fadlallah (1985) \\
\hline & & & Jul/Aug, 1 & Shlesinger \& Loya (1985) \\
\hline Seriatopora caliendrum & $\mathrm{H}$ & B & May-Dec, 8-1 & Rinkevich \& Loya (1979a), Shlesinger \& Loya (1985) \\
\hline Stylophora pustillata & $\mathrm{H}$ & B & Dec-Jul, 1-8 & $\begin{array}{l}\text { Loya (1976), Rinkevich \& Loya }(1979 a, b) \\
\text { Shlesinger \& Loya (1985) }\end{array}$ \\
\hline PORITIDAE & & & & \\
\hline Alveopora daedalea & $\mathrm{H}$ & B & $\mathrm{f}-\mathrm{w}$ & Shlesinger \& Loya (1985) \\
\hline & $0 \mathrm{G}: 15 \mathrm{H}$ & 3B:11S: $1 x$ & & \\
\hline
\end{tabular}

base, spawners outnumber brooders 168:37 (Table 3). Spawning is usually associated with higher fecundity. while brooding produces fewer, larger larvae (Fadlallah 1983).
Szmant-Froelich (1984) proposed that brooders experience the greatest recruitment success in the Caribbean, while spawners (particularly acroporids and poritids) are the more successful recruiters in the Pacific. 
Table 2. Global comparisons of reproductive characteristics in scleractinian corals from the Caribbean, Red Sea, and Pacific regions. See individual entries under regional headings for literature citations

\begin{tabular}{|c|c|c|c|c|c|c|}
\hline & Caribbean & Hawaii & \multicolumn{3}{|c|}{ Pacific } & Red Sea \\
\hline \multicolumn{7}{|l|}{ No. of species for which } \\
\hline $\begin{array}{l}\text { reproductive data are } \\
\text { recorded }\end{array}$ & 19 & 17 & 47 & 145 & 26 & 15 \\
\hline Gonochoric & 6 & 4 & 4 & 34 & 2 & 0 \\
\hline Hermaphroditic & 10 & 6 & 28 & 109 & 24 & 15 \\
\hline Not reported & 3 & 7 & 15 & 2 & 0 & 0 \\
\hline Brood & 12 & 5 & 14 & 8 & 1 & 3 \\
\hline Spawn & 7 & 9 & 28 & 136 & 25 & 11 \\
\hline Not reported & 0 & 3 & 5 & 1 & 0 & 1 \\
\hline No. of species in region & $62^{1}$ & $45^{2}$ & $353^{3}$ & $356^{4}$ & $242^{5}$ & $244^{6}$ \\
\hline \multicolumn{7}{|c|}{$\begin{array}{l}\text { Sources: (1) Goreau \& Wells (1967), (2) Jokiel (1987), (3) R. Randall, pers. comm., (4) Willis et al. (1985) (5) Veron (1985), (6 } \\
\text { Sheppard (1987) }\end{array}$} \\
\hline
\end{tabular}

Table 3. Summary of reproductive mode for 210 species of scleractinian corals for which data are available. Entries reflect cumulative counts, omitting repeats for species found in 2 or more regions

\begin{tabular}{|lrcc|}
\hline & Hermaphroditic & Gonochoric & Unknown \\
\hline Spawn & 131 & 37 & 0 \\
Brood & 11 & 7 & 19 \\
Unknown & 1 & 2 & $390+$ \\
\hline
\end{tabular}

She also noted that Caribbean Porites, which are brooders, have small adult colony size, while the spawning gonochoric Pacific Porites are large and long-lived. Pacific Porites species which brood form small, encrusting colonies ( $P$. stephensoni [as P. haddoni], Marshall \& Stephenson 1933; P. murrayensis, Kojis \& Quinn 1981 b; P. brighami, Hunter \& Hodgson unpubl.).

Timing, seasonality, synchrony, and periodicity

Sexual reproduction in corals may occur yearly (Willis et al. 1985, Babcock et al. 1986), seasonally (Rinkevich \& Loya 1979a, b, Szmant 1986, Tomascik \& Sander 1987), monthly (Marshall \& Stephenson 1933, Kawaguti 1941, Atoda 1947 a, Richmond \& Jokiel 1984), or not at all (Grigg et al. 1981, Richmond 1985, Richmond \& Hunter unpubl.). Annual multispecies synchronous spawning has been observed for over 140 species on the Great Barrier Reef (Harrison et al. 1984, Willis et al. 1985, Babcock et al. 1986, Harrison pers. comm.), while asynchrony is exhibited among coral species in the Central Pacific, Hawaii, Okinawa, and the Red Sea (Table 1). Synchronous development and release of gametes among individuals in a population are important to maximize the probability of successful cross (and/or self) fertilization. Conversely, a presumed advantage to multiple spawnings or planulations is to minimize the effects of a single catastrophic event on an individual's or population's reproductive success

Temperature, photoperiod, and noctumal illumination all appear to be important in providing temporal cues which may allow synchrony within populations (Kojis \& Quinn 1981 a, Jokiel et al. 1985, Willis et al. 1985, Hunter 1989). The expanding database suggests that the degree of multispecies synchrony may be correlated with the annual temperature range experienced by the corals (Shlesinger \& Loya 1985, Babcock et al. 1986). Annual variation in seawater temperature is $2.2^{\circ} \mathrm{C}$ in the Central Pacific (Guam; Emery 1962), $3.2^{\circ} \mathrm{C}$ in the Caribbean (Barbados; Tomascik \& Sander 1987), $4.0^{\circ} \mathrm{C}$ in Hawail (Oahu; Jokiel 1985), $6.0^{\circ} \mathrm{C}$ in the Red Sea (Eilat; Rinkevich \& Loya $1979 \mathrm{~b}), 9.8^{\circ} \mathrm{C}$ in Okinawa (Nakamura 1984), and $12.0^{\circ} \mathrm{C}$ on the Great Barrier Reef (Magnetic Island; Babcock et al. 1986). The percentage of reported coral species spawning within the same month and lunar phase for each of these regions is 18 , $26,29,20,65$, and $88 \%$, respectively. Oliver et al. (1989) reported that reproductive seasonality and synchrony among and within coral species distributed from the southern Great Barrier Reef to Papua New Guinea diminishes at lower latitudes. The trend appears to be one of tighter interspecific synchrony with increased temperature range. A similar pattern of less restricted spawning patterns with increasing proximity to the equator was reported for echinoderm species by Pearse (1968).

Differences in reproductive seasonality can occur within a species over its distributional range (Table 4). Most Great Barrier reef species spawn in the austral spring, while spawnings in the Central Pacific, Hawaii, 
Table 4. Global comparisons of reproductive periodicity in coral species which have been reported from 2 or more regions. Abbreviations are for month and lunar day. Month is divided into 8 phases: 1 , new moon, 3, first quarter, 5, full moon, 7, last quarter; 2, 4, 6 and 8 indicate intermediate lunar phases (after Shlesinger \& Loya 1985); w: winter, sp: spring, sr: summer, f: fall, yr: year-round ${ }^{\cdot}$ possibly sterile

\begin{tabular}{|c|c|c|c|c|c|}
\hline & GBR & $\begin{array}{l}\text { Central } \\
\text { Pacific }\end{array}$ & Okinawa & Hawail & Red Sea \\
\hline \multicolumn{6}{|l|}{ ACROPORIDAE } \\
\hline $\begin{array}{l}\text { Acropora cerealis } \\
\text { (as A. hystrix) }\end{array}$ & Nov, 6 & $\begin{array}{l}\text { Jul, } 4-5 \\
\text { sr }\end{array}$ & - & - & - \\
\hline $\begin{array}{l}\text { Acropora cytherea } \\
\text { (as A. corymbosa })^{b}\end{array}$ & Oct/Nov, 6 & Jun/Jul, 1-4 & Jun, 5 & $\cdot$ & - \\
\hline Acropora digitifera & Oct, 6 & Jun, 5 & & & \\
\hline Acropora florida & Nov, 6 & - & Jun, $5 ? 8$ & - & - \\
\hline Acropora formosa & Oct, $6 /$ Nov, 5-6 & - & Jun, $5 ? 8$ & - & - \\
\hline Acropora grandis & Nov, 6 & - & Jun, 5 & - & - \\
\hline Acropora humilis & $\begin{array}{l}\text { Oct, 6/Nov, 5-7 } \\
\text { (spawn) }\end{array}$ & $\begin{array}{l}\text { Jun/Jul, 1-3 } \\
\text { (brood) } \\
\text { Aug, } 7 \\
\text { (spawn) }\end{array}$ & - & $\cdot$ & $\begin{array}{l}\text { May/Jun, } 7 \\
\text { (spawn) }\end{array}$ \\
\hline $\begin{array}{l}\text { Acropora hyacinthus } \\
\text { (as A. surculosa) }\end{array}$ & Oct/Nov. 5-6 & Jul, 7-8 & Jun, 5 & - & Jul, 3 \\
\hline Acropora latistella & Sep/Oct, 6 & - & Jun, 7 & - & - \\
\hline 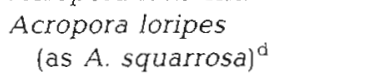 & Nov/Dec, 6 & Jul, 2-3 & & & \\
\hline Acropora micropthalma & Oct/Nov, 6 & - & Jun, $5 / 7$ & - & - \\
\hline Acropora nasuta & Nov, $6-7$ & $\begin{array}{l}\text { Jul, } 7-8 \\
\text { Aug, 6-7 }\end{array}$ & - & - & - \\
\hline Acropora nobilis & Oct/Nov, 6 & & Jun, 5 & & \\
\hline $\begin{array}{c}\text { Acropora robusta } \\
\text { (as A. smithi) }\end{array}$ & Nov, 6 & Jul, $4-5$ & & & \\
\hline $\begin{array}{l}\text { Acropora selago } \\
{\text { (as A delicatula })^{1}}^{\text {as }}\end{array}$ & Nov, 6 & $\cdot$ & & & \\
\hline Acropora tenuis & $\begin{array}{l}\text { Oct, } 6 \\
\text { Nov, } 5-6\end{array}$ & Jul, 6 & Jun, 5 & - & - \\
\hline Acropora valida & $\begin{array}{l}\text { Oct, } 6-7 \\
\text { Nov, } 5-6\end{array}$ & Jul, $7-8$ & Jun, 5 & $\cdot$ & - \\
\hline (as A. variabilis $)^{\mathrm{g}}$ & $\mathrm{sp}-\mathrm{sr}$ & $\mathrm{sr}$ & & & \\
\hline Montipora aequituberculata & Oct, 6 & - & Jun, 5-6 & - & - \\
\hline Montipora digitata & Oct/Nov, 5 & - & Jun, 5-6 & - & - \\
\hline Montipora turgescens & Nov, 6 & - & Jun, 5 & - & - \\
\hline \multicolumn{6}{|l|}{ FAVIIDAE } \\
\hline Favia favus & Nov, 6 & - & - & - & $\begin{array}{l}\text { Jun/Jul, 6-7 } \\
\text { Aug, } 6\end{array}$ \\
\hline Favia mathaii & Nov, 5-6 & Jul, 7-8 & - & - & - \\
\hline Favia pallida & Oct/Nov. 6 & - & Jun, 5 & - & - \\
\hline Favia stelligera & Nov, 6 & Jul, 7-8 & - & - & - \\
\hline Favites abdita & Nov, 5-6 & sr & - & - & - \\
\hline Favites chinensis & Nov, 6 & - & $\begin{array}{l}\text { Jun, } 6-7 / \text { Jul, } 7 \\
\text { Aug, } 7\end{array}$ & - & - \\
\hline Favites flexuosa & Nov, 6 & sr & - & - & - \\
\hline Goniastrea aspera & $\begin{array}{l}\text { Oct, 5-7 } \\
\text { Nov, 5-6 } \\
\text { (spawns) }\end{array}$ & $\begin{array}{l}\text { Oct/Nov, } 1 \\
\text { (broods?) }\end{array}$ & Jun/Jul, 5-6 & - & - \\
\hline Goniastrea edwardsl & Nov, 6 & sr & - & - & - \\
\hline Goniastrea retiformis & Nov, 6 & Jul, 1-2 & - & - & Jul/Aug, 7 \\
\hline Leptoria phrygia & Nov, 6 & Jul, 7-8 & - & - & - \\
\hline $\begin{array}{l}\text { a Synonymized with } A \text {. cerea } \\
\text { "Synonymized with } A \text {. cythe } \\
\text { "Synonymized with A. hyaci } \\
\text { "Synonymized with A. loripe } \\
\text { "Synonymized with A. robus } \\
\text { Synonymized with A. selago } \\
\text { g Synonymized with A. valid }\end{array}$ & $\begin{array}{l}\text { y Veron \& Wallac } \\
\text { by Veron \& Walla } \\
\text { s by Veron \& Wal } \\
\text { Veron \& Wallace } \\
\text { yeron \& Wallace } \\
\text { Veron \& Wallace } \\
\text { Veron \& Wallace }\end{array}$ & $\begin{array}{l}(1984) \\
\text { e }(1984) \\
\text { ace }(1984) \\
(1984) \\
(1984) \\
1984) \\
1984)\end{array}$ & & & \\
\hline
\end{tabular}


Table 4 (continued)

\begin{tabular}{|c|c|c|c|c|c|}
\hline & GBR & $\begin{array}{l}\text { Central } \\
\text { Pacific }\end{array}$ & Okinawa & Hawaii & Red Sea \\
\hline \multicolumn{6}{|l|}{ FAVIIDAE } \\
\hline Montastrea custa & Nov, 6 & Aug, 6-7 & - & - & - \\
\hline Platygyra daedalea & Oct/Nov, $6-7$ & Jul, $6-7$ & - & - & - \\
\hline Platygyra lamellina & Nov, 6-7 & - & - & - & $\begin{array}{l}\text { Jun/Jul, 1-2 } \\
\text { Aug, } 1\end{array}$ \\
\hline Platygyra pini & Nov, 6 & $\mathrm{sr}$ & Jun, 2 & - & - \\
\hline \multicolumn{6}{|l|}{ FUNGIIDAE } \\
\hline Fungia fungites & Oct/Nov, 6 & $\mathrm{sr}$ & - & - & - \\
\hline $\begin{array}{l}\text { Heliofungia actiniformis } \\
\text { MUSSIDAE }\end{array}$ & Oct/Nov, 5 & Sep-Apr, 1 & - & - & - \\
\hline $\begin{array}{l}\text { Lobophyllia corymbosa } \\
\text { Symphyllia recta }\end{array}$ & $\begin{array}{l}\text { Nov, } 6 \\
\text { Nov, } 6\end{array}$ & - & $\begin{array}{l}\text { Jun, } 6 \\
\text { Jun, } 5\end{array}$ & - & - \\
\hline \multicolumn{6}{|l|}{ OCULINIDAE } \\
\hline Galaxea fascicularis & $\begin{array}{l}\text { Oct/Nov, } 6 \\
\text { (spawns) }\end{array}$ & $\begin{array}{l}\text { Jul, 2-3 } \\
\text { (spawns) } \\
\text { yr } \\
\text { (broods) }\end{array}$ & $\begin{array}{l}\text { Jun/Jul/Aug, 6-7 } \\
\text { (spawns) }\end{array}$ & - & $\begin{array}{l}\text { Jul/Aug/Sep, 6-7 } \\
\text { (spawns) }\end{array}$ \\
\hline \multicolumn{6}{|l|}{ POCILLOPORIDAE } \\
\hline Pocillopora damicornis & $y r, 1-5$ & $\begin{array}{l}\text { yr, } 1 \\
y r, 3-8\end{array}$ & - & yr, $5-3$ & - \\
\hline Pocillopora verrucosa & - & $\begin{array}{l}\text { Jun/Jul, 1-4 } \\
\text { Jan, 1-4 } \\
\text { (broods) }\end{array}$ & - & - & $\begin{array}{l}\text { Jul/Aug, } 1 \\
\text { (spawns) }\end{array}$ \\
\hline Seriatopora hystrix & $s p-s r$ & $\mathrm{sr} / \mathrm{w}, 1-8$ & - & - & - \\
\hline Stylophora pistillata & - & $y r, 5-7$ & - & - & Dec-Jul, 1-8 \\
\hline \multicolumn{6}{|l|}{ PORITIDAE } \\
\hline Porites cylindrica & Nov, 6 & $\cdot$ & - & - & - \\
\hline Porites lobata & $\begin{array}{l}\text { Nov, } 6 \\
\text { Dec, } 1,4-7,8\end{array}$ & Jul, 7-8 & - & Aug, $7-8$ & - \\
\hline Porites lutea & $\begin{array}{l}\text { Jan, 4-8 } \\
\text { Nov-Jan } \\
\text { Nov, 5-7 }\end{array}$ & $\mathrm{sr}$ & - & - & - \\
\hline
\end{tabular}

Okinawa, and the Red Sea occur mostly during summer. For many species, variations in timing and synchrony of spawning have been observed within as well as between the regions summarized in this paper (Table 1). Seasonal (latitudinal) variability within regions (e.g. between Palau, Enewetak and Guam) may be of sufficient magnitude to cause differences in reproductive timing. In addition, spawning may occur at different times for different sections of a single colony, or for different colonies within a population (Willis et al. 1985, Babcock et al. 1986, Hunter \& Richmond unpubl.).

While temperature may be the seasonal cue, nocturnal illumination (lunar phase) may provide the 'fine tuning' for the particular night or nights of gamete or planula release. Both brooding and broadcasting species have been shown to cue on night-time illumination (Jokiel et al. 1985, Hunter 1989). It has also been suggested that tidal regime and onset to darkness may play roles as 'forcing functions', determining the actual time of day when spawning will occur (Harriott 1983 a, Babcock et al. 1986, Hunter 1989).
A distinct lunar planulation cycle was reported for Stylophora pistillata in Palau (Atoda 1947 b), while Red Sea populations of this species show no lunar synchrony (Rinkevich \& Loya 1979 b). Lunar periodicity of planulation was found to differ between populations of Pocillopora damicomis at Enewetak and Hawaii, and within populations in Hawaii (Richmond \& Jokiel 1984). The 'Type $B$ ' morph of $P$. damicornis planulated consistently at first quarter moon, while 'Type $\mathrm{Y}$ ' planulated at last quarter. Van Moorsel (1983) proposed identification of a new species of Agaricia based partly on its distinct planulation schedule.

Differences in timing among allopatric populations of a species may represent adaptations to local environmental parameters and cues. Richmond \& Jokiel (1984) suggested that asynchrony among sympatric populations of an identified 'species' may be the result of the immigration of planulae from one region into another. Reproductive isolation, in this case via temporal mechanisms, allows for divergence and eventual speciation. Such reproductive differences within nominal 




Fig. 1 Acropora tenuis Section through a ripe colony of a simultaneous hermaphrodite, 1 wk prior to spawning. Egg and sperm are found within the same polyp. Egg diameter is ca $0.5 \mathrm{~mm}$

species raise questions concerning taxonomy based on morphological characteristics alone.

\section{Asexual reproduction in corals}

Corals possess the ability to reproduce asexually, which is displayed by a variety of mechanisms. Asexual reproductive processes include formation of 'polypballs' (Rosen \& Taylor 1969), polyp bail-out (Goreau \& Goreau 1959, Sammarco 1982, Richmond 1985), asexual production of planulae (Stoddart 1983), and fragmentation (Highsmith 1982).

Asexual reproduction via fragmentation appears to be important for many coral species, and especially for populations living at the extremes of their physiological limits. Grigg et al. (1981) reported lack of mature gonads in populations of 3 species of Acropora from the Northwest Hawaiian Islands. Reproduction via fragmentation appeared to be the major means of population growth. Likewise, Pocillopora damicornis populations in the eastern Pacific had not produced mature gonads nor planulae during a 2 yr study, yet were the dominant reef species off the coast of Panama (Richmond 1985). Eastern Pacific $P$. damicornis exhibit higher colony growth rates than Central Pacific populations, which enhances population growth via fragmentation (Richmond 1985). High bioerosion rates on corals in the eastern Pacific are proposed as making fragmen- tation important for massive species such as Pavona cactus (Highsmith 1982)

Asexual reproduction of corals is found in all regions covered in this review, but appears to dominate in areas which are marginal for coral growth, including the eastern Pacific (Richmond 1985), the northwest Hawaiian Islands (Grigg et al. 1981), southwestern Australia (Stoddart 1984), and possibly Bermuda (Wyers 1985). Within regions with optimal conditions for coral growth, asexual processes may dominate specific habitats including areas of high wave energy (Tunnicliffe 1981), soft or unconsolidated substrata (Gilmore \& Hall 1976), and stable, undisturbed sites (Hunter 1988, unpubl.). In areas where sexual reproductive processes are prevalent, asexual reproduction may augment recruitment at any time, especially during periods of environmental stress or disturbance (Highsmith et al. 1980).

Enhanced colony growth rate and subsequent fragmentation may result from allocation of energy away from production of sexual products, notably in environments where sexual processes may be physiologically constrained (Richmond $1987 \mathrm{a}$ ). The occurrence of sterile populations has been described for other invertebrate taxa as well (Mileikovsky 1971). Asexual reproduction has the advantages of not requiring a partner, propagating locally adapted genotypes, and providing a refuge-in-size from predation and burial by sediments. 


\section{PLANULA LARVAE}

Coral planulae can result from either internal fertilization and brooding, or external fertilization of spawned gametes and subsequent development outside the parent colony (Harrigan 1972, Babcock \& Heyward 1986). Stoddart (1983) suggested that planulae of Pocillopora damicornis may also be produced asexually, based on similarities of parental and planular multilocus genotypes and adult population structure. Two other species, Tubastrea coccinea and $T$. diaphana, were also found to have planulae with isozyme patterns identical to their broodparents, while planulae of Acropora palifera and Seriatopora hystrix had genotypes consistent with sexual origin (Ayre \& Resing 1986).

The brooded planulae of Pocillopora damicornis contain symbiotic zooxanthellae upon release from the parent, as well as a large quantity of lipid (Fig. 2). With the additional ability to feed while planktonic, these planulae remain competent for over $100 \mathrm{~d}$, a period sufficient to allow dispersal over large distances (Richmond 1981, 1987 a). Planulae resulting from spawned gametes (Fig. 3) may lack zooxanthellae upon fertilization (known exceptions being poritids and Montipora spp., whose eggs contain maternal zooxanthellae), but eventually acquire the algal cells from the environment, usually after settlement and metamorphosis (Babcock 1989). Planulae of Fungia scutaria acquire zooxanthellae after release from the parent colony but before metamorphosis (Krupp 1983).

The spawned larvae of Acropora tenuis do not contain zooxanthellae, and have a shorter competency period (ca 20 d) than the brooded larvae of Pocillopora

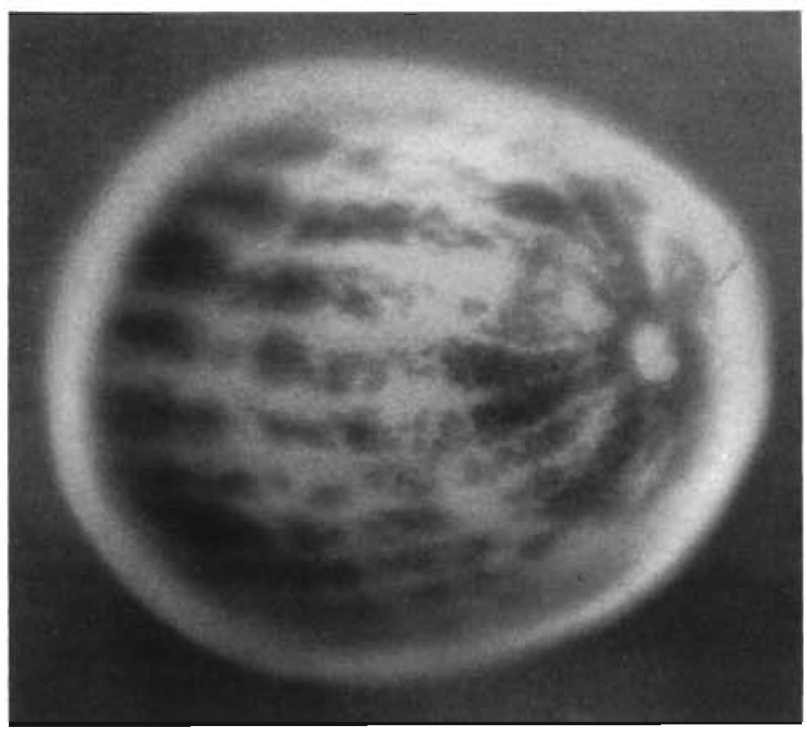

Fig. 2. Pocillopora damicornis. Brooded planula of a coral Note the bands of zooxanthellae, and the oral opening. The larva is ca $1 \mathrm{~mm}$ in length damicornis (Richmond 1989). Larval competency (the ability of larvae to successfully settle and metamorphose) is a major factor affecting the distribution of coral species, particularly for reefs in the eastern Pacific. It has been proposed that the present coral fauna of the eastern Pacific is the result of long-distance dispersal of planulae from Central Pacific stock (Dana 1975, Richmond 1987 b). Some instances of limited distribution patterns and endemism may be the result of abbreviated larval competency periods.

\section{RECRUITMENT}

Reproductive success may best be measured by recruitment. Recruitment of both sexual and asexual propagules is mediated by biotic factors, such as predation and competition, and by abiotic factors such as environmental variability and disturbance. Sexual recruitment of corals is a function of several parameters including the timing of reproduction, competency periods of planula larvae, current regimes, availability of substrata, and densities of predators and competitors (Birkeland et al. 1981, Fitzhardinge 1985, Babcock 1989). Several studies have found evidence for 'open populations' with non-localized sources of coral planulae (Wallace 1985 a, Babcock 1989), but others have suggested that reefs can be self-seeded (Baggett \& Bright 1985, Sammarco \& Andrews 1988, 1989, Andrews et al. 1989). In some areas, juvenile abundance is directly related to adult cover (Bak \& Engel 1979,

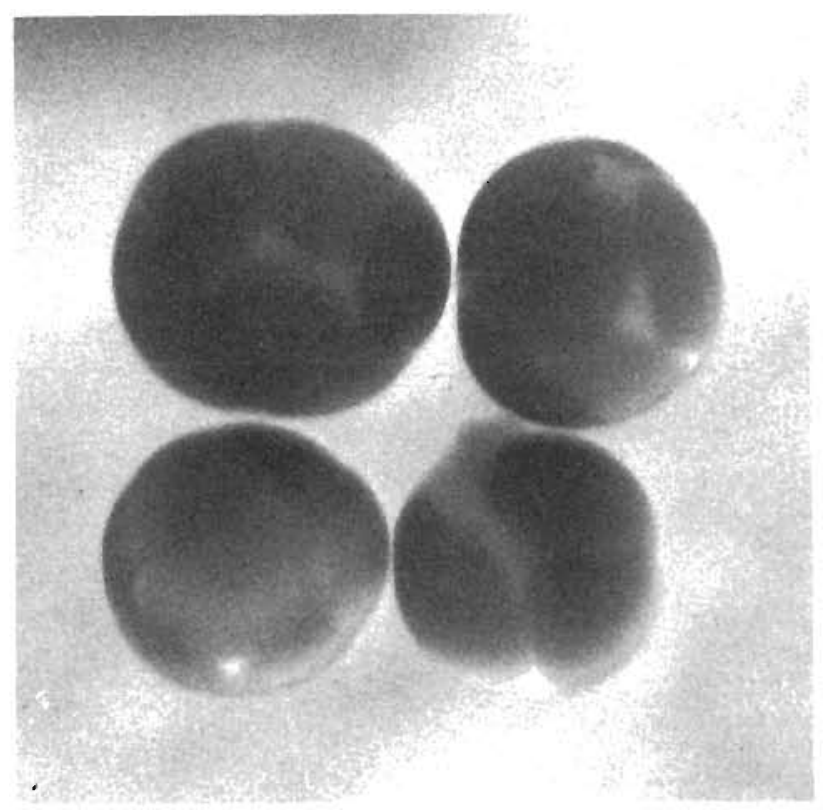

Fig. 3. Acropora tenuis. Spawned clusters of eggs surrounding sperm from a coral. Each cluster contains between 9 and 16 eggs around a single sperm packet Cluster diameters range between 1.3 and $1.6 \mathrm{~mm}$ 
Rylaarsdam 1983), although this is not always the case (Fitzhardinge 1985, Harriott 1985). Relative recruitment rates have been shown to vary from year to year, and among sites on the Great Barrier Reef (Wallace 1985 a).

There appears to be an inverse correlation between success of larval recruitment and propagation by fragmentation in some coral species (Kojis \& Quinn 1981 b, Highsmith 1982, Wallace 1985 b). Sammarco (1985, 1987) reported that, while planular recruitment of Acropora spp. on the Great Barrier Reef is high, juvenile recruits are rare in the Caribbean and populations there are structured primarily by asexual processes. Temporal and regional variations in biotic and environmental factors can cause differences in reproductive and recruitment patterns of coral communities.

The pan-Pacific coral Pocillopora damicornis provides an example of how life history characteristics may vary with respect to local conditions (Richmond 1985, 1987 d). Enewetak Atoll and Hawaii are characterized as having low rates of predation on $P$. damicornis, relatively low seasonal variability, yet relatively high frequencies of mortality-causing disturbances (typhoons and winter storms). P. damicornis colonies planulate monthly throughout the year in Hawail and the Central Pacific, and the oldest colonies observed are estimated to be less than $10 \mathrm{yr}$ old (branch lengths $<20 \mathrm{~cm}$ ). The eastern Pacific of Panama is characterized as having high rates of predation on $P$. damicornis, high levels of seasonal variability, and low frequencies of mortality-causing events. In this area, $P$. damicornis has never been found to release planulae, has a higher colony growth rate, and is the dominant coral in terms of competitive interactions with other corals, with some colonies estimated as over $70 \mathrm{yr}$ old (branch lengths $>240 \mathrm{~cm}$ ).

Theories on the evolution of life history characteristics have been proposed which suggest that under conditions of environmental instability, where lethal disturbances occur at a relatively high rate, formation of large numbers of motile propagules should occur. while under stable conditions, selection will favor clonal (asexual) growth (Williams 1975, MaynardSmith 1978). Likewise, under conditions of low juvenile versus high adult mortality, and relatively low competitive ability versus competitive dominance, selection will favor the sexual mode (Abrahamson 1980, Douglas 1981). Pocillopora damicornis fits these predicted patterns over its distributional range.

\section{RESOURCE MANAGEMENT IMPLICATIONS}

As coral reefs throughout the world are showing signs of degradation, management of reef resources is becoming a growing concern. In the case of corals which spawn during a very brief period each year, the presence of contaminants such as petroleum products, pesticides, herbicides and heavy metals from soils may prevent successful fertilization of eggs by sperm, and hence, severely limit coral recruitment (Richmond unpubl.). It is suggested that pollution levels which may not affect adult coral colonies could still be responsible for the eventual loss of reefs if reproductive processes are disturbed. In Guam and in Okinawa (southern Japan), the peak coral spawning occurs during the rainy season, when levels of coastal contamination via runoff are at their highest (pers. obs.).

With reef degradation and destruction occurring on a global scale, an application of the reproductive data is in the area of reef recovery. Areas of reef which have been destroyed may be re-seeded, and the most efficient means will depend on local conditions. In areas where sedimentation is high, corallivores are present in large numbers, and/or disturbance rate is low, cementing larger numbers of smaller fragments may be more effective based on reproduction and recruitment data. In areas where environmental conditions support sexual reproduction of corals, juvenile mortality is expected to be relatively low, and suitable substratum is available, transplantation of gravid adult colonies into an area may result in highest return of effort. Reef re-seeding is an expensive process (Harriott \& Fisk 1989) and management efforts might best be expended in coral reef protection and conservation.

\section{SUMMARY}

Data on the reproductive biology of $200+$ scleractinian corals indicate several apparent trends. Species which broadcast spawn outnumber those which brood planula larvae. Broadcast spawners typically have limited, annual spawning periods, while most brooders are iteroparous, releasing larvae over a large part of the year. Most corals reported to date are hermaphroditic, some of which have been found to self-fertilize readily in the laboratory; the capacity for selfing is low to nonexistent in other hermaphroditic species. There are reports of coral populations which are apparently sterile, particularly those at the extreme limits of their distributions

There appear to be several geographical trends in coral reproduction. The majority of species on the Great Barrier Reef participate in an annual synchronous spawning event following full moon in the austral spring. In other areas of the Pacific, the Caribbean, and the Red Sea, there is a greater partitioning of spawning periods over more months, days, and lunar phases. Degree of synchrony among species may be related to seawater temperature ranges in each region. 
Mode of reproduction within a given taxa is generally conservative, while timing may be variable within and among species. Differences in reproductive patterns may represent adaptations to local environmental conditions or may represent groups which have not been sufficiently taxonomically differentiated. A point which clearly emerges from the data reviewed in this paper is the need for examination of reproductive characteristics in conjunction with skeletal characters to elucidate what may be real problems in coral taxonomy. Do observed reproductive differences indicate that speciation has occurred among populations? If we accept the biological species definition, it is evident that morphological characters alone may fail to detect valid biological differences. As more studies of reproduction are pursued, resulting information will enable development of a more accurate understanding of the ecology and evolutionary biology of the Scleractinia.

Acknowledgements. We gratefully acknowledge UNESCO for sponsoring the meeting on Interoceanic Comparisons, held at the University of the South Pacific, Suva, Fiji in March, 1986, for which this review was originally prepared. We thank Drs C. E. Birkeland, P. L. Jokiel, R. A. Kinzie, D. A. Krupp and P. W. Sammarco for comments on manuscript drafts, R. Randall for taxonomic identifications, D. Hopper, F. Te, A. Kerr, and A. Goodis for assistance with field and laboratory examination of Guam corals, and Dr K. Yamazato for access to the corals of Okinawa. This is contribution no. 277 of the University of Guam Marine Laboratory.

\section{LITERATURE CITED}

Abe, N. (1937). Postlarval development of the coral Fungia actiniformis var palawensis Doderlein. Palao trop. biol. Stn. Stud. 1: 73-93

Abrahamson, W. G. (1980). Demography and vegetative reproduction. In: Solbrig, O.T. (ed.) Demography and evolution in plant populations. Botanical Monographs 15. Blackwell Scientific Publication, London, p. 89-106

Andrews, J. C., Gay, S. L., Sammarco, P. W (1989). Models of dispersal and recruitment of coral larvae around an isolated reef (Helix Reef) in the central Great Barrier Reef. Proc. 6th Int. Coral Reef Congr., Townsville (in press)

Atoda, K. (1947 a). The larva and postlarval development of some reef-building corals. I. Pocillopora damicornis (Dana). Sci. Rep. Tohoku Univ. 18: 24-47

Atoda, K. (1947 b). The larva and postlarval development of some reef-building corals. II. Stylophora pistillata (Esper.). Sci. Rep. Tohoku Univ. 18: 48-64

Atoda, K. (1951 a). The larva and postlarval development of some reef-building corals. III. Acropora brueggemanni (Brook). J. Morph. 89: 1-13

Atoda, K. (1951 b). The larva and postlarval development of some reef-building corals. IV. Galaxea aspera Quelch. J. Morph. 89: 17-35

Atoda, K. (1951 c). The larva and postlarval development of some reefbuilding corals. IV. Seriatopora hystrix Dana. Sci. Rep. Tohoku Univ. 19: 33-39

Ayre, D. J., Resing, J. M. (1986). Sexual and asexual production of planulae in reef corals. Mar. Biol. 90: 187-190
Babcock, R. C. (1984). Reproduction and distribution of two species of Goniastrea (Scleractinia) from the Great Barrier Reef province. Coral Reefs 2: 187-204

Babcock, R. C. (1989). Fine-scale spatial and temporal patterns in coral recruitment. Proc. 6th Int. Coral Reef Symp., Townsville, in press

Babcock, R. C., Bull, G. D. Harrison, P. L., Heyward, A. J. Oliver, J. K., Wallace, C. C., Willis, B. L. (1986). Synchronous spawning of 105 scleractinian coral species on the Great Barrier Reef. Mar. Biol. 90: 379-394

Babcock, R. C., Heyward, A. J. (1986). Larval development of certain gamete-spawning scleractinian corals. Coral Reefs 5: $111-116$

Baggett, L. S., Bright, T. J. (1985). Coral recruitment at the East Flower Garden Reef. Proc. 5th Int. Coral Reef Cong. Tahiti 4: 379-384

Bak, R. P. M. Engel, M. S. (1979). Distribution, abundance and survival of juvenile hermatypic corals (Scleractinia) and the importance of life history strategies in the parent coral community. Mar. Biol. 54: 341-352

Birkeland, C., Rowley, D., Randall, R. H. (1981). Coral recruitment patterns at Guam. Proc. 4th Int. Coral Reef Symp. Manila, 2: 339-344

Boschma, H. (1929). On the post-larval development of the coral Maendra areolata. Pap. Tortugas Lab. 26: 129-147

Bothwell, A. M. (1981). Fragmentation, a means of asexual reproduction and dispersal in the coral genus Acropora (Scleractinia: Astrocoeniida: Acroporidae) - a preliminary report. Proc. 4th Int. Coral Reef Symp., Manila 2: 137-144

Chornesky, E. A., Peters, E. C. (1987). Sexual reproduction and colony growth in the scleractinian coral Porites astreoides. Biol. Bull. mar. biol. Lab., Woods Hole 161-177

Dana, T.F. (1975). Development of contemporary eastern Pacific coral reefs. Mar. Biol. 33: 355-374

Douglas, D. A. (1981). The balance between vegetative and sexual reproduction of Mimulus primuloides (Scrophulariaceae) at different altitudes in California. J. Ecol. 69: 295-310

Duerden, J. E. (1902). West Indian madreporarian polyps. Mem. natn. Acad. Sci. 8: 401-597

Edmondson, C. H. (1929). Growth of Hawaiian corals. Bull Bernice P. Bishop Mus. 58: 1-38

Edmondson, C. H. (1946). Behavior of coral planulae under altered saline and thermal conditions. Occ. Pap. Bernice P. Bishop Mus. 18: 283-304

Emery, K. O. (1962). Marine geology of Guam. Prof. Pap. U.S. Geol. Surv. 403-B: 1-76

Fadlallah, Y. H. (1983). Sexual reproduction, development and larval biology in scleractinian corals. A review. Coral Reefs 2: $129-150$

Fadlallah, Y. H. (1985). Reproduction in the coral Pocillopora verrucosa on the reefs adjacent to the industrial city of Yanbu, Red Sea, Saudi Arabia. Proc. 5th Int. Coral Reef Symp., Tahiti 4: 314-318

Fitzhardinge, R. (1985). Spatial and temporal variability in coral recruitment in Kaneohe Bay. Proc. 5th Internat. Coral Reef Cong., Tahiti 4: 373-378

Gilmore, M. D., Hall, B. R. (1976). Life history, growth habits, and constructional roles of Acropora cervicornis in the patch reef environment. J. sedim. Petrol. 46: 519-522

Goreau, T. H., Goreau, N. I. (1959). The physiology of skeleton formation in corals. II. Calcium deposition by hermatypic corals under various conditions in the reef. Biol. Bull. mar. biol. Lab., Woods Hole 117: 239-250

Goreau, T. H., Wells, J. M. (1967). The shallow-water scleractinia of Jamaica: revised list of species and their vertical distribution range. Bull. mar. Sci. 17: 442-452 
Grigg, R. W., Wells, J. M. Wallace, C. (1981). Acropora in Hawaii, Part 1: History of the scientific record, systematıcs and ecology. Pacif. Sci. 35: 1-13

Hada, Y (1932). A note of earlier stage of colony formation with the coral Pocillopora caespitosa. Sci. Rep. Tohuku Imp. Univ. 6: 425-431

Harrigan, J. F. (1972). The planula larva of Pocillopora damicornis, lunar periodicity of swarming and substratum selection behavior Ph. D. dissertation, Univ. of Hawail

Harriott, V. J. (1983 a). Reproductive ecology of four scleractinian species at Lizard Island, Great Barrier Reef. Coral Reefs 2: 9-18

Harriott, V. J. (1983 b). Reproductive seasonality, settlement, and post-settlement mortality of Pocillopora damicornis (Linnaeus), at Lizard Island, Great Barrier Reef. Coral Reefs 2: 151-157

Harriott, V. J. (1985). Recruitment patterns of scleractinian corals at Lizard Island, Great Barrier Reef. Proc. 5th Int Coral Reef Cong., Tahiti 4: 367-372

Harriott, V. J., Fisk, D. A. (1989). Coral transplantation as a reef management option. Proc. 6th Int. Coral Reef Symp. Townsville (in press)

Harrison, P. L. (1985). Scxual characteristics of scleractinian corals: systematic and evolutionary implications. Proc. 5th Int. Coral Reef Symp., Tahiti 4: 337-342

Harrison, P. L. (1989). Pseudo-gynodieocy: an unusual breeding system in the scleratinian coral Galaxea fascicularis Proc. 6th Int. Coral Reef Symp., Townsville (in press)

Harrison, P. L., Babcock, R. C., Bull, G. D., Oliver, J. K., Wa] lace, C. C., Willis, B. L. (1984). Mass spawning in tropical reef corals. Science 223: 1186-1189

Heyward, A. J. (1985). Sexual reproduction in five species of the coral Montipora. Tech. Rep. Hawaii Inst. Mar. Biol. 37 $170-178$

Heyward, A. J. (1989). Reproductive status of some Guam corals. Micronesica 21. 272-274

Heyward, A. J., Babcock, R. C. (1986). Self- and cross-fertilization in scleractinian corals. Mar Biol. 90: 191-195

Heyward, A. J., Collins, J. D. (1985). Growth and sexual reproduction in the scleractinian coral Montipora digitata (Dana). A.ust. J. mar Freshwat. Res. 36: 441-446

Heyward, A. J., Yamazato, K., Yemin, T., Minei, M. (1987) Sexual reproduction of corals in Okinawa. Galaxea 6 : $331-343$

Highsmith, R. C. (1982). Reproduction by fragmentation in corals. Mar Ecol. Prog. Ser 7: 207-226

Highsmith, R. C., Riggs, A. C., D'Antonio, C. M. (1980). Survival of hurricane-generated coral fragments and a disturbance model of reef calcification/growth rates. Oecologia (Berl.) 46: 322-329

Hunter, C. L. (1988). Genotypic diversity and population structure of the Hawaiian reef coral, Porites compressa. Ph. D dissertation, Univ. of Hawaii

Hunter, C. L. (1989). Environmental cues controlling spawning in two Hawaiian corals: Montipora verrucosa and $M$ dilatata. Proc. 6th Int. Coral Reef Symp., Townsville (in press)

Jokiel, P. L. (1985). Lunar periodicity of planula release in the reef coral Pocillopora damicornis in relation to various environmental factors. Proc. 5th Int. Coral Reef Symp., Tahiti 4: 307-312

Jokiel, P. L. (1987). Ecology, biogeography and evolution of corals in Hawall. Trends Ecol. Evol. 2 (7): 179-182

Jokiel, P. L., Ito, R. Y., Lu, P. M. (1985). Night irradiance and synchronization of lunar spawning of larvae in the reef coral Pocillopora damicornis (Linnaeus). Mar Biol. 88: 167-174 Kawaguti, S. (1941). On the physiology of reef corals. $V$
Tropisms of coral planulae considered as a factor of distribution on the reef. Palao trop. biol. Stn Stud. 2: 319-328

Kojis, B. L., Quinn, N. J. (1981 a). Aspects of sexual reproduction and larval development in the shallow water hermatypic coral Goniastrea australensis (Edwards and Haime 1857). Bull. mar. Sci. 31: 558-573

Kojis, B. L., Quinn, N. J. (1981 b). Reproductive strategies in four species of Porites (Scleractinia). Proc. 4th Int. Coral Reef Symp., Manila 2: 145-151

Kojis, B. L., Quinn, N. J. (1982). Reproductive ecology of two Faviid corals (Coelenterata: Scleractinia). Mar Ecol. Prog. Ser. 8: 251-255

Krupp, D. A. (1983). Sexual reproduction and early development of the solitary coral Fungia scutaria (Anthozod: Scleractinia). Coral Reefs 2: 159-164

Loya, Y (1976). The Red Sea coral Stylophora pistillata is an rstrategist. Nature, Lond. 259: 478-480

Marshall, S. M. Stephenson, T. A. (1933). The breeding of reef animals. Part I. The corals. Scient. Rep. Gt Barrier Reef Exped. 3: 219-245

Mavor, J. W. (1915). On the development of the coral Agaricia fragilis Dana. Proc. Am. Acad. Arts Sci. 51: 485-511

Maynard-Smith, J. (1978). The evolution of sex. Cambridgo University Press, London

Mileikovsky, S. A. (1971). Types of larval development in marine bottom invertebrates, their distribution and ecological significance: a re-evaluation. Mar Biol. 10: 193-213

Nakamura, S. (1984). Record of air temperature, surface water temperature and chlorinity at Sesoko in 1983. Galaxea 3 : 105

Oliver, J., Babcock, R., Harrison, P., Willis, B. (1989). The geographic extent of mass coral spawning: clues to proximate and ultimate causal factors. Proc. 6th Int. Coral Reef Symp., Townsville (in press)

Pearse, J. S. (1968). Patterns of reproduction in four species of Indo-Pacific echinoderms. Proc. Indian Acad. Sci. 67 . $247-267$

Reed, S. A. (1971). Techniques for raising the planula larvae and newly settled polyps of Pocillopora damicornis. In: Lenhoff, H. M., Muscatine, L., Davis, L. V. (eds.) Experimental coelenterate biology. Univ. Hawaii Press

Richmond, R. H. (1981). Energetic considerations in the dispersal of Pocillopora damicornis planulae. Proc. 4th Int. Coral Reef Symp., Manila 2: 153-156

Richmond, R. H. (1985). Variations in the population biology of Pocillopora damicornis across the Pacific Ocean. Proc. 5th Int. Coral Reef Cong., Tahiti 6: 101-106

Richmond, R. H. (1987 a). Energetic relationships and biogeographical differences among fecundity, growth, and reproduction in the reef coral, Pocillopora damicornis. Bull. mar Sci. 41 (2): 595-604

Richmond, R. H. (1987 b). Energetics, competency, and longdistance dispersal of planula larvae of the coral Pocillopora damicornis. Mar. Biol. 93: 527-533

Richmond, R. H. (1989). Competency and dispersal of spawned versus brooded coral planula larvae. Proc. 6th Int. Coral Reef Cong., Townsville (in press)

Richmond, R. H., Jokiel, P. L. (1984). Lunar periodicity in larva release in the reef coral Pocillopora damicomis at Enewetak and Hawaii. Bull. mar. Sci. 34: 280-287

Rinkevich, B., Loya, Y (1979 a). The reproduction of the Red Sea coral Stylophora pistillata. I. Gonads and planulae Mar Ecol. Prog. Ser 1. 133-144

Rinkevich, B., Loya, Y (1979 b). The reproduction of the Red Sea coral Stylophora pistillata. II. Synchronization in breeding and seasonality of planula shedding. Mar. Ecol Prog. Ser 1. 145-152 
Rosen, B. R., Taylor, J. D. (1969). Reef coral from Aldabra: new mode of reproduction. Science 166: 119-120

Rylaarsdam, K.W (1983). Life histories and the abundance patterns of colonial corals on Jamaican reefs. Mar. Ecol. Prog. Ser 13: 249-260

Sammarco, P. W. (1982). Polyp bail-out: an escape response to environmental stress and a new means of reproduction in corals. Mar Ecol. Prog. Ser 10: 57-65

Sammarco, P. W. (1985). The Great Barrier Reef vs. the Caribbean: comparisons of grazers, coral recruitment patterns and reef recovery. Proc. 5th Int. Coral Reef Cong., Tahiti 4 : 391-397

Sammarco, P. W. (1987). A comparison of ecological processes on coral reefs of the Caribbean and the Great Barrier Reef. In: Birkeland, C. (ed.) Comparison between Atlantic and Pacific tropical marine coastal ecosystems: community structure, ecological processes, and productivity. Univ. So. Pacif., Suva, Fiji, 1986. UNESCO Rep. mar. Sci. 46: 127-166

Sammarco, P. W. Andrews, J. C. (1988). Localized dispersal and recruitment in Great Barrier Reef corals: The Helix Experiment. Science 239: 1422-1424

Sammarco, P. W., Andrews, J. C. (1989). The Helix Experiment: differential localised dispersal and recruitment patterns in Great Barrier Reef corals. Limnol. Oceanogr. (in press)

Sheppard, C. R. C. (1987). Coral species of the Indian Ocean and adjacent seas: a synomized compilation and some regional distribution patterns. Atoll Res. Bull. 307: 1-32

Shlesinger, Y., Loya, Y (1985). Coral community reproductive patterns: Red Sea versus the Great Barrier Reef. Science 228: 1333-1335

Stimson, J. S. (1978). Mode and timing of reproduction in some common hermatypic corals of Hawaii and Enewetak. Mar. Biol. 48: 173-184

Stoddart, J. A. (1983). The asexual production of planulae in the coral Pocillopora damicornis. Mar. Biol. 76: 279-284

Stoddart, J. A. (1984). Genetical structure within populations of the coral Pocillopora damicornis. Mar. Biol. 81 19-30

Szmant, A. M. (1986). Reproductive ecology of Caribbean reef corals. Coral Reefs 5: 43-53

Szmant-Froelich, A. (1984). Reef coral reproduction: diversity and community patterns. In: Advances in reef science.

This review was presented by Dr. P. W. Sammarco,

Townstille, Australia
Joint Meeting Intl. Soc. for Reef Studies and Atlantic Reef Committee. University of Miami، Miami, p. 122-123

Szmant-Froelich, A., Reutter, M., Riggs, L. (1985). Sexual reproduction of Favia fragum (Esper): lunar patterns of gametogenesis, embryogenesis and planulation in Puerto Rico. Bull. mar. Sci. 37: 880-892

Tomascik, T., Sander, F. (1987). Effects of eutrophication on reef-building corals. III. Reproduction of the reef-building coral Porites porites. Mar Biol. 94: 77-94

Tunnicliffe, $V$ (1981). Breakage and propagation of the stony coral Acropora cervicornis. Proc. natn. Acad Sci. USA 78: $2427-2431$

Van Moorsel, G. W. N. M. (1983). Reproductive strategies in two closely related stony corals (Agaricia, Scleractinia). Mar. Ecol. Prog. Ser 13: 273-283

Vaughan, T W (1910). The recent Madreporaria of southern Florida. Yb. Carnegie Instn. Wash. 7: 135-144

Veron, J.E.W. (1985). Corals of Australia and the IndoPacific. Angus and Robertson, London

Veron, J. E. W., Wallace, C. C. (1984). Scleractinia of Eastern Australia, Part V, Family Acroporidae. A. I. M. S (Australian Institute of Marine Science, Townsville) Monogr Ser Vol. 6, p. $1-485$

Wallace, C. C. $(1985 \mathrm{a})$. Four years of juvenile coral recruitment to five reef front sites. Proc. 5th Int. Coral Reef Cong., Tahiti 4: 385-390

Wallace, C. C. (1985 b). Reproduction, recruitment and fragmentation in nine sympatric species of the coral genus Acropora. Mar. Biol. 88: 217-233

Williams, G. C. (1975). Sex and evolution. Princeton University Press, New Jersey

Willis, B. L., Babcock, R. C., Harrison, P. L., Oliver, T K. (1985). Patterns in the mass spawning of corals on the Great Barrier Reef from 1981 to 1984. Proc. 5th Int. Coral Reef Cong., Tahiti $4: 343-348$

Wyers, S. C. (1985). Sexual reproduction of the coral Diploria strigosa (Scleractinia, Favidae) in Bermuda: research in progress. Proc. 5th Int. Coral Reef Cong., Tahiti 4: 301-306

Yamazato, K., Oshiro, M. Oshiro E. (1975). Reproductive biology of a scleractinian coral, Goniopora queenslandiae decima. Proc. 13th Pac. Sci. Congr. 1: 135 (Abstr.)

Manuscript first received: January 8, 1988

Revised version accepted: October 18, 1989 